\title{
Blob velocity scaling in diverted tokamaks: A comparison between theory and simulation
}

F

Cite as: Phys. Plasmas 26, 032302 (2019); https://doi.org/10.1063/1.5080675

Submitted: 10 November 2018 . Accepted: 31 January 2019 . Published Online: 01 March 2019

Paola Paruta, C. Beadle (D), P. Ricci (D), and C. Theiler (D)

\section{COLLECTIONS}

F This paper was selected as Featured
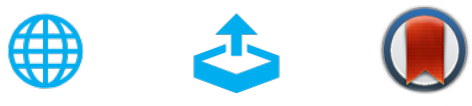

\section{ARTICLES YOU MAY BE INTERESTED IN}

Self-consistent simulation of transport and turbulence in tokamak edge plasma by coupling SOLPS-ITER and BOUT++

Physics of Plasmas 26, 012508 (2019); https://doi.org/10.1063/1.5084093

Full-f gyrokinetic simulation of turbulence in a helical open-field-line plasma

Physics of Plasmas 26, 012307 (2019); https://doi.org/10.1063/1.5074179

Wavelet methods for studying the onset of strong plasma turbulence

Physics of Plasmas 25, 122310 (2018); https://doi.org/10.1063/1.5062853 


\title{
Blob velocity scaling in diverted tokamaks: A comparison between theory and simulation $\odot$
}

\author{
Cite as: Phys. Plasmas 26, 032302 (2019); doi: 10.1063/.5080675 \\ Submitted: 10 November 2018 - Accepted: 31 January 2019 . \\ Published Online: 1 March 2019
}

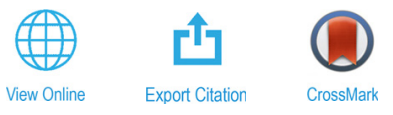

Paola Paruta, C. Beadle, (D) P. Ricci, (D) and C. Theiler (D)

\section{AFFILIATIONS}

Ecole Polytechnique Fédérale de Lausanne (EPFL), Swiss Plasma Center (SPC), CH-1015 Lausanne, Switzerland

\begin{abstract}
The present work uses the results of a fluid full-turbulence 3D simulation of the tokamak periphery to present the first selfconsistent analysis of the radial velocity scaling of plasma blobs in a diverted geometry. A diverted double-null configuration is considered, and the blob motion is studied using a pattern recognition algorithm. The velocity obtained from the simulation results is compared to an analytical scaling accounting for the presence of the X-point. Agreement is found between numerical and analytical results.
\end{abstract}

Published under license by AIP Publishing. https://doi.org/10.1063/1.5080675

\section{INTRODUCTION}

The dynamics in the periphery of magnetic fusion devices is characterised by the presence of blobs. These are coherent structures of enhanced plasma density with respect to the background, spatially localised in the plane perpendicular to the magnetic field and elongated in the parallel direction. ${ }^{1}$ Because of their shape, blobs are also known as filaments. Blobs detach from the main plasma and move radially outwards, making turbulence at the edge of fusion devices intermittent and significantly contributing to the radial transport mechanisms in the SOL. Blobs have been observed in tokamaks (e.g., in the Caltech Research Tokamak, ${ }^{2}$ Alcator C-Mod, ${ }^{3}$ JET, ${ }^{4}$ JT-60U, ${ }^{5}$ Tore Supra, ${ }^{6}$ and $\mathrm{TCV}^{7}$ ), stellarators (e.g., in the W7-AS stellarator ${ }^{8}$ and in $\mathrm{TJ}-\mathrm{K}^{9}$ ) reversed field pinches, ${ }^{10}$ and basic plasma devices (e.g., in LAPD ${ }^{11}$ and in TORPEX ${ }^{12}$ ). They can lead to enhanced intermittent heat flux on the main vessel wall, possibly damaging radio frequency antennas and wall tiles and causing sputtering of impurities. ${ }^{13-15}$

It is generally believed that blobs are the result of the nonlinear saturation of interchange-like instabilities in the edge, with the density fluctuation sheared apart by the $\mathbf{E} \times \mathbf{B}$ velocity and detached from the main plasma, as observed in $\mathrm{JET}^{16}$ and in TORPEX, ${ }^{12}$ and as described by 2D fluid models, e.g., Ref. 17. Once detached from the main plasma, blobs move radially outwards. An extensive review of the literature on blob motion can be found in Ref. 1. The radial motion results from the vertical charge separation inside the blob stemming from the effect of the magnetic gradient and curvature drifts. The charge separation leads to an electric field and its associated $\mathbf{E} \times \mathbf{B}$ drift that causes the blob to move radially outwards. This basic mechanism of radial motion is confirmed by a series of blob studies conducted on the TORPEX device, ${ }^{18,19}$ by experiments in limited and diverted plasmas on $\mathrm{TCV},{ }^{20}$ and by numerical simulations of seeded blobs (see, e.g., Refs. 21-24). Considering a selfconsistent simulation of a TCV discharge in a limited configuration, in Ref. 25, a pattern-recognition algorithm for blob tracking showed good agreement of the blob velocity with the theoretical scalings.

In the present work, we investigate for the first time the velocity scaling of blobs self-consistently generated in a simulation of SOL plasma turbulence in diverted configurations. In previous studies, the effect of the X-point on blob motion has been investigated using simulations of a single seeded blob; ${ }^{26}$ for this purpose, the BOUT $++\operatorname{code}^{27}$ was used, to reproduce the experimental work of Ref. 19 on TORPEX. In addition, the magnetic shear effect of blobs has been studied as a proxy for the X-point in Refs. 28 and 29. Only very recently, the study of blob motion has been approached by using 3D full-turbulent self-consistent simulations in diverted configurations. The results of a full 3D turbulent simulation with the XGC1 gyrokinetic code of a DIII-D H-mode discharge have been used to carry out an initial investigation of the blob properties. ${ }^{30}$ In the present work, we extend the use of the pattern recognition algorithm introduced in Ref. 25 to analyse the blob motion in a full SOL turbulent simulation in a double-null configuration. The simulation is carried out with the GBS code. $\mathrm{GBS}^{31-33}$ is a 3D code that simulates the plasma turbulent dynamics in the 
tokamak periphery by evolving the two-fluid drift reduced Braginskii's equations. ${ }^{34,35}$ In the past few years, GBS has helped investigating plasma dynamics in limited tokamaks, for example, by providing predictions of the SOL width. ${ }^{36}$ Recently, GBS capabilities have been extended to the simulation of diverted scenarios, ${ }^{33}$ by abandoning the use of flux coordinates, which present a singularity at the X-point.

The results from a GBS simulation in the diverted doublenull configuration are here compared with a theoretical scaling developed to predict the blob velocity in the presence of an X-point. ${ }^{37}$ As pointed out also by a recent experimental study on ASDEX Upgrade ${ }^{38}$ and in simulations, ${ }^{39}$ collisionality can affect the blob velocity scaling. Our results focus on the high collisionality regime, and depending on the blob size, we identify the polarization current or the parallel current as balance mechanisms to the interchange drive. Our simulation results are in good agreement with the theoretical scalings.

This paper is organised as follows: Leveraging previous derivations by Myra et al., ${ }^{37}$ we provide the analytical scaling to estimate the velocity of blobs in diverted configurations in Sec. II. Then, Sec. III presents the GBS simulation results obtained in the double-null configuration. Blobs are detected and tracked to determine their velocity, size, and collisionality, by using the blob tracking technique presented in Sec. IV. Finally, the simulation results of the blob radial velocity are compared with the analytical scaling in Sec. $V$ which are followed by conclusions.

\section{BLOB VELOCITY ANALYTICAL SCALING IN THE PRESENCE OF AN X-POINT}

Analytical predictions of the blob radial velocity can be obtained by using simplified 2D two-fluid models, describing the plasma dynamics in the plane perpendicular to the magnetic field. These models usually consider the continuity equation, charge conservation, and a closure for the parallel current. Examples can be found in Refs. 13, 18, and 40. The most investigated of such analytical 2D models to account for the effect of an X-point on blob transport is the two-region model. ${ }^{37}$ The two-region model separates the upstream and the divertor regions, labelled as regions 1 and 2, respectively (see Fig. 1). In the upstream region, the unfavourable curvature of the magnetic field leads to the formation of an electric dipole that provides most of the drive for the blob radial motion. In the divertor region, the magnetic flux expansion causes the blob to elongate in one direction and squeeze in the other (to guarantee mass conservation). The stretching of the blob facilitates the damping of the blob charge separation by cross-field currents.

By following the calculation in Ref. 37, we retain the corrections due to the blob density, blob ellipticity, and magnetic field line length difference between the two regions in the evaluation of the blob velocity. We derive the two-region model in the Appendix. In this section, we present the final results of our calculation and leave the details to the Appendix.

The two-region model allows the identification of four different blob motion regimes, ${ }^{37}$ which correspond to four different mechanisms to balance the curvature drive in region 1. In the sheath connected regime, denoted as $\mathrm{C}_{\mathrm{s}}$, the curvature drive is balanced by the current flowing to the sheath. In the ideal

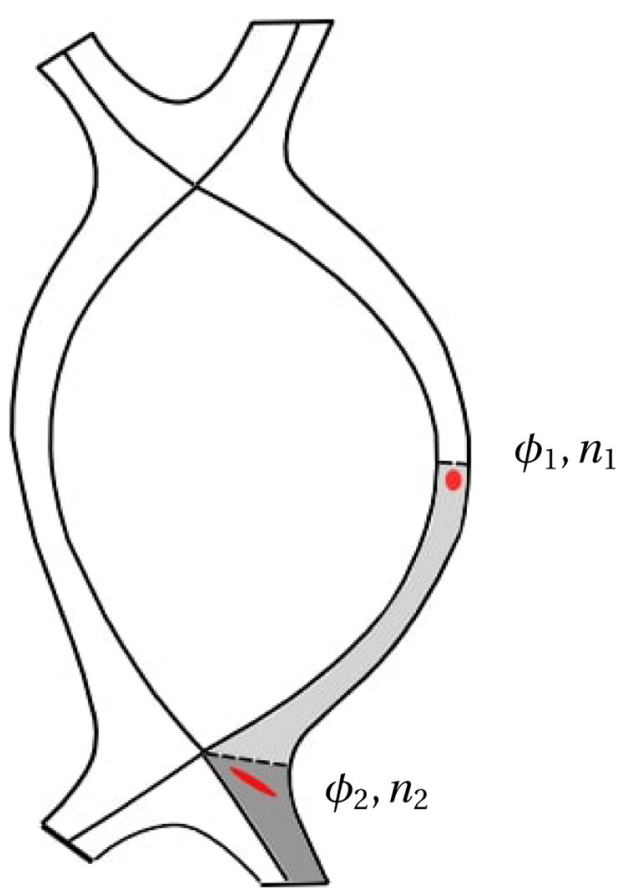

FIG. 1. Schematic illustration of the two region model. Region 1 (light gray) corresponds to the outboard low field side, where the curvature drive $\beta$ is active, and extends from the midplane to the $X$-point region. Here, the flux expansion is maximal and causes the blobs to elongate and tilt due to field line mapping, disconnecting region 1 from the divertor, i.e., region 2 in darker gray, characterised by the current to the sheath.

interchange mode regime, $\mathrm{C}_{i}$, the ion polarisation current in region 2, due to the fanning of the flux surfaces, balances the drive. In the resistive ballooning regime, $\mathrm{RB}$, the ion polarisation current in region 1 dominates. Finally, in the resistive X-point regime, RX, the parallel current flowing between the two regions is the key damping mechanism. Each regime is characterised by a different blob velocity to size scaling.

We find that the regimes are defined by the values of the following parameters:

$$
\begin{gathered}
\Lambda=\frac{\nu^{e / i} L_{1}^{2}}{\rho_{s} \Omega_{e} L_{2}}, \\
\Theta=\hat{a}^{\frac{5}{2}}=\left(\frac{a_{b}}{a^{*}}\right)^{\frac{5}{2}} .
\end{gathered}
$$

The collisionality parameter $\Lambda$ in Eq. (1) can be interpreted as the ratio between the resistivity in region 1 and the sheath resistivity. If $\Lambda$ is high, region 1 is decoupled from the sheath. In Eq. (1), $\nu_{1}^{e / i}$ is the electron to ion collision frequency in region 1 , present in Ohm's law, while $\rho_{\mathrm{s}}=\sqrt{\mathrm{T}_{e} / m_{i}} / \Omega_{i}$ is the ion sound gyroradius. The electron gyro-frequency $\Omega_{e}$ is defined as $\Omega_{e}=e \mathrm{~B} / \mathrm{m}_{e}$ ( $e$ is the electron charge, $m_{e}$ is the electron mass, and $B$ is the magnetic field strength at the blob location in region 1). We note that the two-region model is derived in the isothermal limit; hence, $\mathrm{T}_{e}$ at the target is considered to be the same as $\mathrm{T}_{e}$ in 
region 1, with no distinction between the blob and background temperature. In Eq. (1), the parallel lengths of the magnetic field line in regions 1 and 2 are $L_{1}$ and $L_{2}$, respectively.

The parameter $\Theta$ and blob normalised size $\hat{a}$ in Eq. (2) are given by the ratio between the physical blob size $a_{b}$ and the reference size $a^{\star}$. The expressions in physical units for the blob size $a_{b}$ and the reference size $a^{*}$ as derived in the Appendix (removing the large aspect ratio approximation) are

$$
\begin{gathered}
a_{b}=\left(\frac{2 a_{y}}{\pi}\right)^{\frac{4}{5}} a_{x}^{\frac{1}{5}} \\
a^{*}=\rho_{s}\left(\frac{2 L_{2}^{2}}{\rho_{s} R}\right)^{\frac{1}{5}}\left[\frac{\Delta_{x} n_{1}}{n_{0,1}}\right]^{\frac{1}{5}} .
\end{gathered}
$$

With respect to Myra et al., ${ }^{37}$ Eq. (3) provides an expression for $a_{b}$ which differentiates the radial (or poloidal) blob size $a_{x}$ (or $a_{y}$ ). More precisely, the radial direction $x$ is the $\nabla \psi$ direction orthogonal to the flux surfaces and $y$ indicates the binormal direction orthogonal to both $\nabla \psi$ and magnetic field versor $\mathbf{b}$. The term within square brackets in Eq. (4), not appearing in Ref. 37, accounts for the effect of blob density and blob ellipticity. The parameter $n_{0,1}$ in Eqs. (4)-(6) corresponds to the mean between the maximal blob density value at the midplane and the background equilibrium density value. $\Delta_{X} n_{1}$ and $a_{x}$ are introduced to approximate the density gradient in the radial direction as a ratio between a density difference and a radial length, i.e., $\partial_{x} n_{1}$ $\sim \Delta_{x} n_{1} / a_{x}$. Reference 37 introduced a practical way to visualise the four regimes in terms of $\Lambda$ and $\Theta$, as well as their transition thresholds (see Fig. 2). Each regime presents a different velocity scaling in terms of the normalised velocity

$$
\hat{v}=\frac{v_{x}}{v^{*}},
$$

with

$$
v^{*}=c_{s, 1}\left(\frac{\rho_{s}^{2} L_{2}}{R^{3}}\right)^{\frac{1}{5}}\left[8 \frac{\left(\delta n_{1}\right)^{5}}{\left(\Delta_{x} n_{1}\right)^{2} n_{0,1}^{3}}\left(\frac{\pi a_{x}}{2 a_{y}}\right)^{2}\right]^{\frac{1}{5}} .
$$

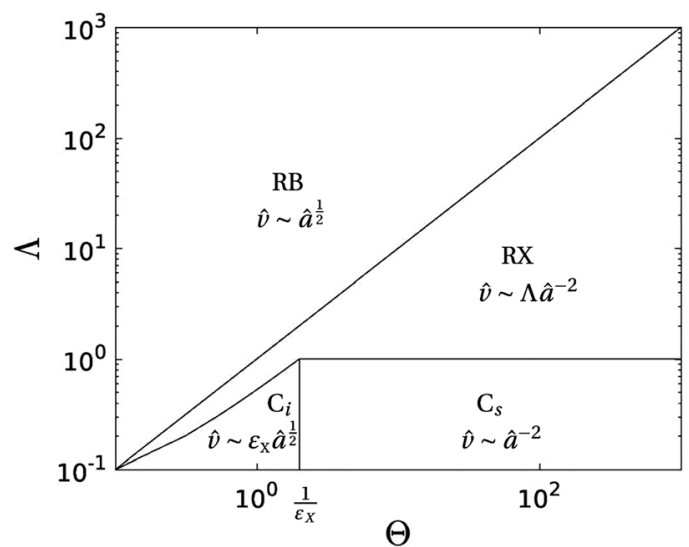

FIG. 2. Characterisation of blob regimes in the $(\Lambda, \Theta)$ plane and correspondent velocity to size scaling, as derived in Ref. 37 from the two region model.
Here, $\delta n_{1}$ is the amplitude of the density fluctuation above $n_{0,1}$ and $a_{y}$ is the half width half maximum (HWHM) blob poloidal size, i.e., half of the poloidal blob size measured half way between the blob density maximum and the background density value (corresponding to the $n_{0,1}$ threshold). The major radius $R$ has to be taken at the blob location in region 1 , around midplane, as it results from approximating the magnetic field curvature at the origin of the drive in region 1.

\section{GBS SIMULATION IN THE DOUBLE-NULL CONFIGURATION}

The present section presents the physical model behind the GBS code, as well as the results of a simulation run in the double-null configuration which will be used for our study. GBS implements a two-fluid model based on the drift-reduced Braginskii's set of equations. ${ }^{35}$ The interaction between the plasma and the wall is described by the magnetic pre-sheath boundary conditions. ${ }^{41}$ The code uses toroidal coordinates to allow for the simulation of diverted configurations. Reference 33 reported on the implementation and verification of the version of GBS used for the present study.

\section{A. Physical model}

The drift-reduced approximation of the Braginskii equations $^{34}$ in the study by Zeiler et $a .^{35}$ relies on the assumption that turbulence in the periphery of a tokamak device occurs on a time scale considerably longer than the gyro-motion $\left[\partial_{t} \ll \Omega_{i}\right.$ $\left.=e \mathrm{~B} /\left(m_{i} c\right)\right]$ and on a length scale larger than the ion gyro-radius $\rho_{i}$. As a result, the velocity perpendicular to the magnetic field line can be described as the sums of the $\mathbf{E} \times \mathbf{B}$, diamagnetic velocity, and ion-polarisation drifts. The cold-ion version of the drift-reduced Braginskii's set of equations used for this work can be written as

$$
\frac{\partial n}{\partial \mathrm{t}}=-\frac{\rho_{\star}^{-1}}{\mathrm{~B}}[\phi, n]+\frac{2}{\mathrm{~B}}\left[\mathrm{C}\left(p_{e}\right)-n \mathrm{C}(\phi)\right]-\nabla_{\|}\left(n v_{\| e}\right)+\mathrm{S}_{n}+\mathrm{D}_{n} \nabla_{\perp}^{2} n,
$$

$$
\begin{aligned}
\frac{\partial v_{\| e}}{\partial \mathrm{t}}= & -\frac{\rho_{\star}^{-1}}{\mathrm{~B}}\left[\phi, v_{\| e}\right]-v_{\| e} \nabla_{\|} v_{\| e}+\frac{m_{i}}{m_{e}} \\
& \times\left(\nu \mathrm{J}_{\|}+\nabla_{\|} \phi-\frac{1}{n} \nabla_{\|} p_{\| e}-0.71 \nabla_{\|} \mathrm{T}_{e}\right) \\
& +\frac{4}{3 n} \frac{m_{i}}{m_{e}} \eta_{0, e} \nabla_{\|}^{2} v_{\| e}+\mathrm{D}_{v_{\| e}} \nabla_{\perp}^{2} v_{\|\| e},
\end{aligned}
$$

$$
\begin{aligned}
\frac{\partial v_{\| i}}{\partial \mathrm{t}}= & -\frac{\rho_{\star}^{-1}}{\mathrm{~B}}\left[\phi, v_{\| i}\right]-v_{\| i} \nabla_{\|} v_{\| i}-\frac{1}{n} \nabla_{\|}\left(p_{e}\right)+\frac{4}{3 n} \eta_{0, i} \nabla_{\|}^{2} v_{\| i} \\
& +\mathrm{D}_{v_{\|}} \nabla_{\perp}^{2} v_{\| i},
\end{aligned}
$$

$$
\begin{aligned}
& \frac{\partial \mathrm{T}_{e}}{\partial \mathrm{t}}=-\frac{\rho_{\star}^{-1}}{\mathrm{~B}}\left[\phi, \mathrm{T}_{e}\right]-v_{\| e} \nabla_{\|} \mathrm{T}_{e}+\frac{4}{3} \frac{\mathrm{T}_{e}}{\mathrm{~B}}\left[\frac{1}{n} \mathrm{C}\left(p_{e}\right)+\frac{5}{2} \mathrm{C}\left(\mathrm{T}_{e}\right)-\mathrm{C}(\phi)\right] \\
&+\frac{2}{3} \mathrm{~T}_{e}\left[0.71 \nabla_{\|} v_{\| i}-1.71 \nabla_{\|} v_{\| e}+0.71\left(v_{\| i}-v_{\| e}\right) \frac{\nabla_{\|} n}{n}\right] \\
&+\mathrm{S}_{\mathrm{T}_{e}}+\chi_{\perp, e} \nabla_{\perp}^{2} \mathrm{~T}_{e}+\chi_{\|, e} \nabla_{\|}^{2} \mathrm{~T}_{e} \\
& \frac{\partial \omega}{\partial \mathrm{t}}=-\frac{\rho_{\star}^{-1}}{\mathrm{~B}}[\phi, \omega]-v_{\| i} \nabla_{\|} \omega+\frac{\mathrm{B}^{2}}{n} \nabla_{\|} \mathrm{J}_{\|}+\frac{2 \mathrm{~B}}{n} \mathrm{C}\left(p_{e}\right)+\mathrm{D}_{\omega} \nabla_{\perp}^{2} \omega \\
& \nabla_{\perp}^{2} \phi=\omega .
\end{aligned}
$$


In Eqs. (7)-(12), all variables are dimensionless, and in the following, we use a tilde to denote physical variables, unless specified otherwise. We define the plasma density $n=\tilde{n} / n_{0}$, the electron temperature $\mathrm{T}_{e}=\tilde{\mathrm{T}}_{e} / \mathrm{T}_{e 0}$, the electrostatic potential $\phi=e \tilde{\phi} / \mathrm{T}_{e 0}$, the electron parallel velocity $v_{\| e}=\tilde{v}_{\| e} / c_{s 0}$, the ion parallel velocity $v_{\| i}=\tilde{v}_{\| i} / c_{s 0}$, and the vorticity $\omega=\tilde{\omega} e \rho_{\mathrm{s} 0}^{2} / \mathrm{T}_{e 0}$ with $n_{0}, T_{e 0}, c_{s 0}=\sqrt{T_{e 0} / m_{i}}$, and $\rho_{\mathrm{s} 0}=c_{\mathrm{s} 0} / \Omega_{\mathrm{ci}}$ the reference density, temperatures, sound velocity, and ion sonic Larmor radius expressed in physical units. The time is defined as $t=\tilde{t} c_{s 0} / R_{0}$, where $R_{0}$ is the major radius at the magnetic axis, in physical units. The electron pressure is $p_{e}=n \mathrm{~T}_{e}$. The current is $\mathrm{J}_{\|}=n\left(v_{\| i}-v_{\| e}\right)$. In our simulation, the values of the dimensionless parameters appearing in Eqs. (7)-(11) are $\rho_{*}=\rho_{\mathrm{s} 0} / \mathrm{R}_{0}=1 / 500$ (normalised ion sonic Larmor radius), $\nu=e n_{0} R_{0} /\left(m_{i} c_{s 0} \sigma_{i}\right)=1$ (normalised Spitzer resistivity), $\eta_{0, e}=5 e-3, \eta_{0, e, i}=1, \chi_{\| e}=1$, and $\chi_{\perp, e}=2$. To reduce the computational cost of the simulation, we use $m_{i} / m_{e}=200$. The minor radius is $a=127 \rho_{\mathrm{s} 0}$. Additionally, small numerical diffusion terms of the type $D_{f} \nabla_{\perp}^{2} f$ are added for numerical stability (in this simulation, $D_{f}=2$ for all fields). In the density and temperature equations, $S_{n}$ and $S_{T_{e}}$ denote source terms that mimic the outflow of plasma and heat from the core.

The dimensionless spatial operators appearing in Eqs. (7)-(12) are the parallel gradient $\nabla_{\|} f=R_{0} \mathbf{b} \cdot \tilde{\nabla} f$, the parallel diffusion operator $\nabla_{\|}^{2} f=\nabla_{\|}\left(\nabla_{\|} f\right)$, the Poisson brackets $[\phi, f]$ $=\rho_{s 0}^{2} \mathbf{b} \cdot(\tilde{\nabla} \phi \times \tilde{\nabla} f)$, the curvature operator $C(f)=R_{0} \rho_{s 0} \tilde{B} / 2$ $(\tilde{\nabla} \times(\mathbf{b} / \tilde{\mathrm{B}})) \cdot \tilde{\nabla}$, and the perpendicular diffusion operator $\nabla_{\perp}^{2}=\rho_{\mathrm{s} 0}^{2} \tilde{\nabla} \cdot((\mathbf{b} \times \tilde{\nabla} f) \times \mathbf{b})$. Here, $f$ indicates one of the dimensionless fluid quantities $\left(n, \mathrm{~T}_{e, i}, v_{\| e, i}, \omega, \phi\right)$, while $\tilde{\mathrm{B}}$ and $\mathbf{b}=\tilde{\mathbf{B}} / \tilde{\mathrm{B}}$ are the norm and the versor of the magnetic field. In $(r, \theta, \varphi)$ toroidal coordinates, we assume an axisymmetric magnetic field of the form

$$
\tilde{\mathbf{B}}=\mathrm{R}_{0} \mathrm{~B}_{0} \nabla \varphi+\nabla \varphi \times \nabla \tilde{\psi}(\tilde{r}, \theta),
$$

with $B_{0}$ the magnetic field at the magnetic axis and $\tilde{\psi}$ the magnetic poloidal flux. Under the assumption of small inverse aspect ratio $\varepsilon=a / R_{0}$ and large safety factor $q$ and using Eq. (13), the differential operator expressions in $(r, \theta, \varphi)$ toroidal coordinates are

$$
\begin{gathered}
\nabla_{||} f=\frac{\mathrm{B}_{0}}{\left|\mathrm{~B}_{0}\right|} \frac{\partial f}{\partial \varphi}+\frac{a}{\rho_{\mathrm{s} 0}} \partial_{\hat{r}} \psi \frac{1}{r} \frac{\partial f}{\partial \theta}-\frac{a}{\rho_{\mathrm{s} 0}} \frac{1}{\hat{r}} \partial_{\theta} \psi \frac{\partial f}{\partial r}, \\
{[\phi, f]=\frac{1}{r} \frac{\mathrm{B}_{0}}{\left|\mathrm{~B}_{0}\right|}[\phi, f]_{r, \theta},} \\
\mathrm{C}(f)=\frac{\mathrm{B}_{0}}{\left|\mathrm{~B}_{0}\right|}\left(\sin \theta \frac{\partial f}{\partial r}+\frac{\cos \theta}{r} \frac{\partial f}{\partial \theta}\right), \\
\nabla_{\perp}^{2} f=\frac{\partial^{2} f}{\partial r^{2}}+\frac{1}{r^{2}} \frac{\partial^{2} f}{\partial \theta^{2}},
\end{gathered}
$$

where two different dimensionless forms of the radial coordinate appear: $\hat{r}=\tilde{r} / a$ is used in relation to $\psi$ derivatives and $r=\tilde{r} / \rho_{\mathrm{s} 0}$ in relation to $f$ derivatives. The dimensionless poloidal flux $\psi$ is defined as $\psi=\tilde{\psi} /\left(a^{2} \mathrm{~B}_{0}\right)$. The details of the derivation of the differential operators can be found in Ref. 33. Since the physical model in Eqs. (7)-(12) considers the electrostatic case, the equilibrium magnetic field is unperturbed throughout the simulation and $\partial_{\hat{r}} \psi, \partial_{\theta} \psi / \hat{r}$ are given as input to the simulation. For this study, $\psi$ is chosen to describe a double-null configuration

$$
\begin{aligned}
\psi(\hat{r}, \theta)= & S\left(\log (\hat{r}-c)+\frac{1}{2} I \log \left((\hat{r}-c)^{2}+4-4(\hat{r}-c) \sin \theta\right)\right. \\
& \left.+\frac{1}{2} I \log \left((\hat{r}-c)^{2}+4+4(\hat{r}-c) \sin \theta\right)\right),
\end{aligned}
$$

with $S=0.03, I=10$, and $c=0.9$.

The GBS domain corresponds to a torus with a hollow poloidal cross section (as the tokamak core is not simulated), as it is represented in Fig. 3. At the numerical wall $r=r_{\max }$, the magnetic pre-sheath boundary conditions developed by Ref. 41 are considered (neglecting correction terms linked to $f$ derivatives along the wall)

$$
\begin{aligned}
v_{\|, i} & = \pm \sqrt{\mathrm{T}_{e}} \\
v_{\|, e} & = \pm \sqrt{\mathrm{T}_{e}} \max \left\{\exp \left(\lambda-\frac{\phi}{\mathrm{T}_{e}}\right), \exp (\lambda)\right\} \\
\partial_{r} \phi & =\mp \sqrt{\mathrm{T}_{e}} \partial_{r} v_{\|, i} \\
\partial_{r} n & =\mp \frac{n}{\sqrt{\mathrm{T}_{e}}} \partial_{r} v_{\|, i} \\
\omega & =-\left(\partial_{r} v_{\|, i}\right)^{2} \mp \sqrt{\mathrm{T}_{e}} \partial_{r r}^{2} v_{\|, i} \\
\partial_{r} \mathrm{~T}_{e} & =0
\end{aligned}
$$

where $\lambda=3$. The plus/minus indicates whether the magnetic field points towards (top sign) or out from the wall (bottom sign) and coincides with the sign of $\mathrm{B}_{r}$, the radial component of $\mathbf{B}$. At the wall location where $B_{r}=0$, the boundary condition for $v_{\| i}$ jumps from $-\sqrt{\mathrm{T}_{e}}$ to $+\sqrt{\mathrm{T}_{e}}$ (or vice-versa), and a similar discontinuity arises for $v_{\| e}$. A smoothing function is applied in the vicinity of $B_{r}$ to avoid such discontinuity (see Ref. 33). At the inner radial boundary, $r=r_{\text {min }}$, we use an ad hoc set of boundary conditions, i.e., $\partial_{r} f=0$ for all fields $f$, except for $\omega$ and $\phi$, for which we impose $\omega=0$ and $\phi=\lambda \mathrm{T}_{e}$. The presence of the source

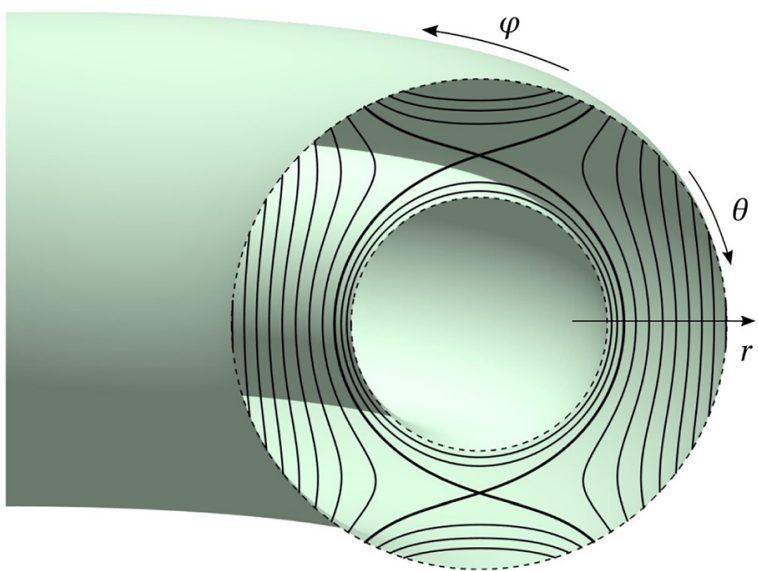

FIG. 3. Visualisation of the GBS computational domain and toroidal coordinates $(r$, $\theta, \varphi)$ used to implement the double-null configuration in Eq. (18). 
of plasma and temperature at $r>r_{\text {min }}$ helps decouple the inner ad hoc boundary conditions to the edge and SOL dynamics.

We note that a more complete version of the equations implemented in GBS for diverted magnetic configurations is presented in Ref. 33. The GBS version for a limited configuration additionally solves neutral dynamics and can be run without the Boussinesq approximation for the vorticity and with electromagnetic effects. $^{32}$

We finally remark that the drift-reduced Braginskii's equations are solved using a numerical scheme based on a fourth order finite difference algorithm with the explicit Runge-Kutta fourth order method for the time stepping. ${ }^{33}$

\section{B. Simulation results}

The simulation is run on a numerical grid $\mathrm{N}_{r} \times \mathrm{N}_{\theta} \times \mathrm{N}_{\varphi}$ $=156 \times 450 \times 80$, with a time step $\Delta t=2 \times 10^{-5}$.

After an initial transient, the simulation reaches a quasisteady state where a strong blob activity is present on the lowfield side (LFS) of the device, leading to the transport of the plasma out-flowing from the tokamak closed flux surface region to the far SOL. Typical snapshots in this turbulent regime for different plasma quantities appearing in the drift-reduced Braginskii's equations are shown in Fig. 4. Here, the turbulence level is considerably lower in the HFS (high-field side) with respect to the LFS, due to the favourable magnetic curvature in the region, resulting in a SOL width 6 times smaller at the HFS.
This asymmetry is present also in single null scenarios, but it is accentuated when using double null configurations. ${ }^{42,43}$ The density $n$ (top-left plot) peaks in the closed flux surface region, around the plasma source location. The blobs at the equatorial midplane of the LFS present a mushroom shape typical of high collisionality regimes. ${ }^{37}$ At the blob locations, fluctuations in the electric potential $\phi$ (top-right plot) reveal the presence of the dipolar structure responsible for the blob motion. The GBS physical model allows $\mathrm{T}_{e}$ to vary (bottom-left plot in Fig. 4). A temperature difference between blobs and the background increases the drive for the blob radial motion through the enhancement of the $\mathrm{C}\left(p_{e}\right)$ term in the vorticity equation and causes the blob to rotate due to $\mathbf{E} \times \mathbf{B}$ drift $^{13}$ because $\phi \propto \mathrm{T}_{e}$ in the SOL in the sheath-limited regime. The rotation of the blob dipole can reduce its outwards radial motion. We assume that these effects are negligible (or balance) and use the two-region model, which is isothermal, to analyse the results. The parallel current $J_{\|}$in Fig. 4 exhibits turbulent behaviour at the LFS where the blobs are located.

\section{BLOB TRACKING TECHNIQUE AND EVALUATION OF BLOB PARAMETERS}

The analysis of blob motion is performed in a time window of $73 R_{0} / c_{s 0}$ time units during the quasi-steady state. To detect blobs in the GBS simulation, we use a pattern recognition algorithm similar to the one presented in Ref. 25. The analysis is carried out in the poloidal plane since the large inverse aspect ratio
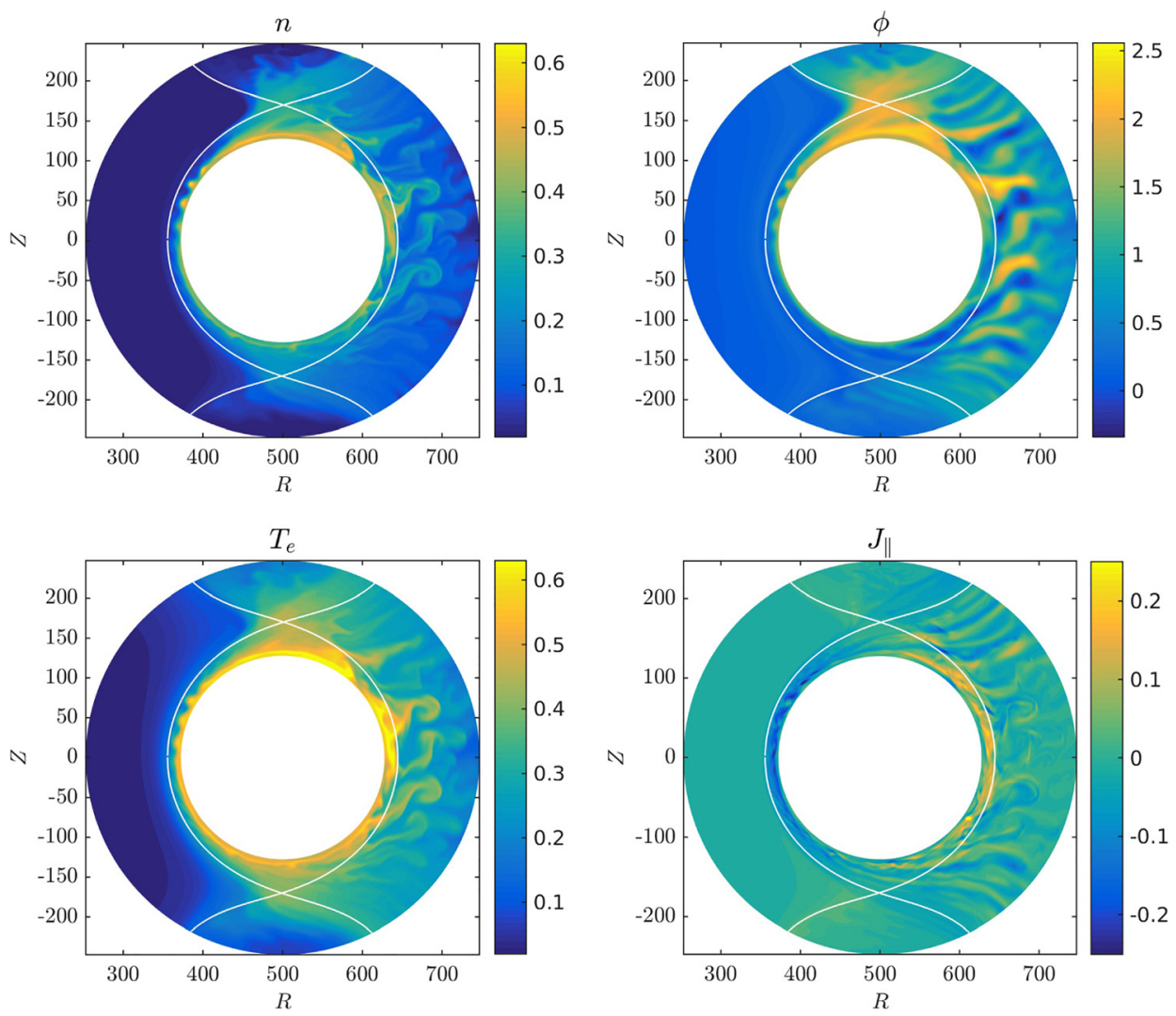

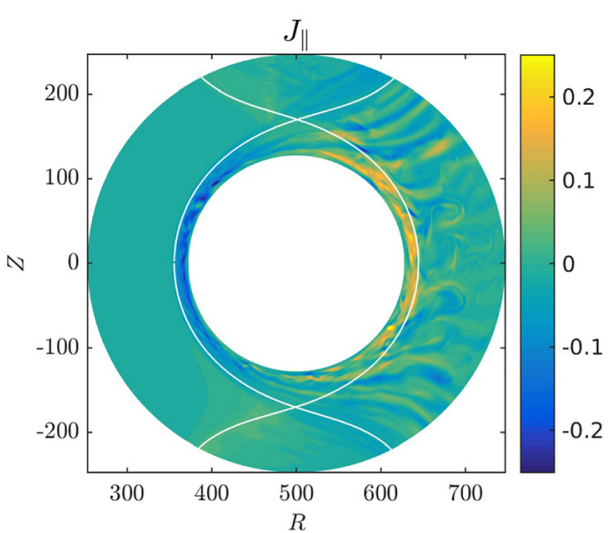

FIG. 4. From left to right, top to bottom, typical snapshots of plasma density, electric potential, electron temperature, and plasma current in the GBS double-null simulation considered for our blob analysis (the separatrix is traced by the white line). 
and large safety factor assumptions allow us to approximate the plane perpendicular to $\mathbf{b}$ with the poloidal plane. We define as a blob a structure of enhanced density (at least 2.5 times the fluctuation level) which moves coherently (i.e., it exists for $\Delta t>0.2$ ). More precisely, blobs are detected from the simulation results as follows. We first identify the regions $\Omega_{b \text {,high }}$ with the density larger than the average density, $n_{b g}$, by 2.5 times the fluctuation level, $\sigma_{n}$, that is

$$
n(r, \theta, t)>n_{b g}+2.5 \sigma_{n}
$$

We remark that the average background density is computed by time and toroidally averaging the density during the quasisteady state, $n_{b g}(r, \theta)=\langle n\rangle_{t, \varphi}$, and the standard deviation is defined as $\sigma_{n}(r, \theta)=\sqrt{\left\langle\left(n\left(r, \theta, \varphi_{k}, t_{m}\right)-n_{b g}(r, \theta)\right)^{2}\right\rangle_{t, \varphi}}$. Once the regions $\Omega_{b \text {,high }}$ where Eq. (20) is satisfied are detected, a pattern recognition algorithm groups the points that are connected and therefore belong to and form the same blob. A blob is then tracked from one time frame to the next by checking whether there is spatial overlapping between $\Omega_{b \text {,high }}$ belonging to two subsequent time frames. Splitting and merging of blobs are also allowed by checking if two blobs end up corresponding to one single blob in the following time frame or vice-versa. The blob detection is carried out in one poloidal plane, and we analyse only the blobs detected around the outboard midplane. This also avoids counting the same blob twice, when the blob extends over the magnetic field by more than one toroidal turn.

Having detected the blobs, we determine their size and velocity. The algorithm described above is efficient in tracking blobs, but it often underestimates the blob size, as it only detects the high density peak of a blob, which is shown by the striped region in Fig. 9. In order to determine the blob size in a way consistent with the analytical two-region model, one needs to detect the region corresponding to the density fluctuation above the half maximum, $n_{0}$. This corresponds to the colored region that we will refer to as $\Omega_{b}$ in Fig. 9. To determine $\Omega_{b}$, we take an area $\Omega_{b, \text { ext }}$ larger than $\Omega_{b, \text { high }}$ by $\sim 30 \rho_{\mathrm{s} 0}$ in every direction and re-define the blob as the set of connected $(r, \theta)$ points in $\Omega_{b, \text { ext }}$ for which

$$
n(r, \theta, t)>n_{0}=n_{b g}+\delta n=n_{b g}+\max _{\Omega_{b . h . h h}(t)} \frac{n-n_{b g}}{2} .
$$

We note that the poloidal radius of $\Omega_{b}$ is the half-width halfmaximum (HWHM) of the blob density perturbation, corresponding to $a_{y}$ of the two-region model (see Fig. 9). The blob HWHM is commonly used to indicate the blob size in blob studies. $^{25,44}$

The blob detection algorithm also verifies the presence of sufficient overlapping in the subsequent time frames

$$
\frac{\left\|\Omega_{b}\left(t_{m}\right) \cap \Omega_{b}\left(t_{m+1}\right)\right\|}{\left\|\Omega_{b}\left(t_{m}\right)\right\|}>0.8
$$

as well as

$$
\left|\frac{\left\|\Omega_{b}\left(t_{m}\right)\right\|-\left\|\Omega_{b}\left(t_{m+1}\right)\right\|}{\left\|\Omega_{b}\left(t_{m}\right)\right\|}\right|<0.2,
$$

to assess that the blob size does not change abruptly. If the blob domain $\Omega_{b}$ changes considerably from one time frame to the next, we consider them as two different blobs. The threshold coefficients 0.8 and 0.2 in the double-null case are chosen so that the blobs have size and shape that are continuous enough, without incurring excessive splitting.

In order to compare the two-region model in Sec. II with the simulation results, we estimate $a_{x}, a_{y}, \Lambda, \Theta=\hat{a}^{5 / 2}$ $=\left(a_{b} / a^{*}\right)^{5 / 2}$, and $\hat{v}=v_{x} / v^{*}$ from the blob parameter in region 1. We focus on blobs detected in the proximity of the midplane, and therefore, we require the blob center of mass to be at most $50 \rho_{\mathrm{s} 0}$ away from midplane, $-50<\mathrm{Z}_{\mathrm{CM}}<50$, with the center of the mass location $\left(R_{C M}, Z_{C M}\right)$ defined as

$$
\mathrm{R}_{\mathrm{CM}}=\frac{\langle\mathrm{R} n(\mathrm{R}, \mathrm{Z})\rangle_{\Omega_{b}}}{\langle n\rangle_{\Omega_{b}}}, \quad \mathrm{Z}_{\mathrm{CM}}=\frac{\langle\mathrm{Zn}(\mathrm{R}, \mathrm{Z})\rangle_{\Omega_{b}}}{\langle n\rangle_{\Omega_{b}}} .
$$

In the proximity of the midplane, $x$ and $y$ directions correspond approximately to the radial and vertical directions, $R$ and $Z$. Therefore, we approximate the blob radii $a_{x}=a_{\mathrm{R}}$ and $a_{y}=a_{Z}$ to correspond to half of the extension of $\Omega_{b}$ along the $\mathrm{R}$ and $\mathrm{Z}$ directions.

To have a better estimate of the blob size, we use the average between the top $10 \%$ of the $\Delta \mathrm{Z}$ and $\Delta \mathrm{R}$ values for each blob, where $\Delta Z(\Delta R)$ is the vertical (radial) extension of the blob area at a given $R(Z)$.

To estimate the quantity $n_{0,1}$ in Eqs. (4) and (6), we take the minimum value of $n$ in $\Omega_{b}$, as suggested by Fig. 9, averaging over the lowest $10 \%$ density values. To compute the density perturbation $\Delta_{x} n_{1}$ that we use to approximate $\partial_{x} n_{1}$, we look at the maximal blob density difference along $R$, for every fixed $Z$ with $(R, Z)$ $\in \Omega_{b}$, which we denote $\left.\Delta n_{b}\right|_{z}$, and we take the average of the top $10 \%$ values. Analogously, to compute $\delta n_{1}$ in Eq. (6), we consider the largest blob density difference along $Z$. Note that if the background density value is constant in the radial direction (across the blob domain), then $\delta n_{1}=\Delta_{x} n_{1}$ and the two estimates coincide. Finally, we compute the Larmor radius $\rho_{\mathrm{s}}$ which in GBS dimensionless units corresponds to $\sqrt{\mathrm{T}_{e}}$, using a similar technique to the one used to evaluate $n_{0,1}$.

The radial velocity $v_{x}=v_{R}$ (in $c_{s 0}$ units) is computed by tracking the radial center of the mass location $R_{C M}$ during a blob lifetime

$$
v_{\mathrm{R}}\left(\mathrm{t}_{i}\right)=\frac{\mathrm{R}_{\mathrm{CM}}\left(\mathrm{t}_{i+1}\right)-\mathrm{R}_{\mathrm{CM}}\left(\mathrm{t}_{i}\right)}{\mathrm{t}_{i+1}-\mathrm{t}_{i}} \rho_{*}^{-1}
$$

where $t_{i}$ is the snapshot time (in the present study, $t_{i+1}-t_{i}$ $=0.05)$.

In the considered double-null configuration, the magnetic field line length in the upstream region $1, L_{1}$, is approximately $2 /$ 3 of the magnetic field line length from the target to the midplane $L_{\|}$(in the proximity of the separatrix). This can be computed numerically as

$$
L_{\|}=\frac{1}{2} \int d l_{\|}=\frac{1}{2} \int \rho_{*} \frac{\sqrt{\mathrm{B}_{\text {pol }}^{2}+\mathrm{B}_{\text {tor }}^{2}}}{\mathrm{~B}_{\text {pol }}} d s,
$$

where $B_{\text {tor }}^{2}=B_{\varphi} B^{\varphi}=1, B_{p o l}^{2}=B_{\theta} B^{\theta}+B_{r} B^{r}=\varepsilon^{2}\left(\left(\partial_{\hat{r}} \psi\right)^{2}+\left(\partial_{\theta} \psi / \hat{r}\right)^{2}\right)$, with $\varepsilon$ being the inverse aspect ratio, and the integral from the lower to the upper strike point is performed along a flux surface. 


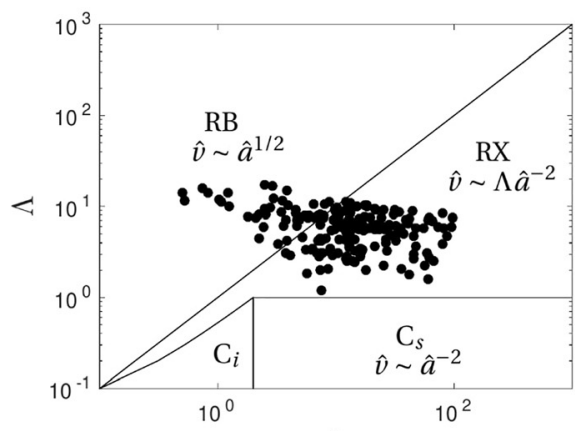

$\Theta$

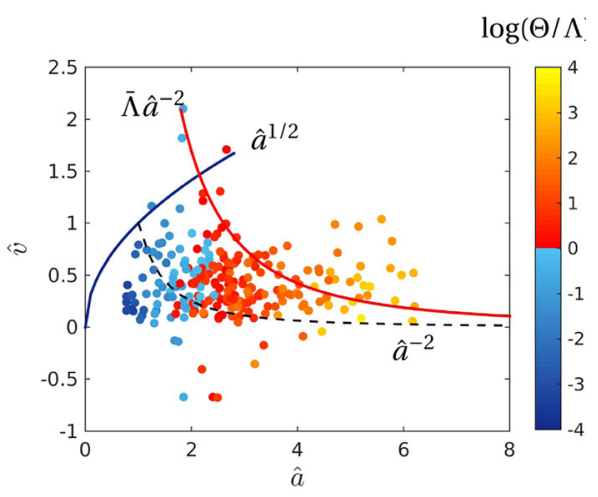

FIG. 5. Characterisation of blob regimes in the $(\Theta, \Lambda)$ plane (left panel). The blobs belong to the resistive ballooning (RB) and resistive $X(R X)$ regimes. The normalised blob velocity $\hat{v}=v_{z} / v^{*}$ as a function of the normalised size $\hat{a}=a_{b} / a^{*}$ (right panel). Good agreement with the analytical scalings of $R B$ and $R X$ regimes (blue and red solid lines), and very different behaviour with respect to the $\mathrm{C}_{s}$ sheath connected regime (black dashed line) is shown. The color-scheme indicates $\log (\Theta / \Lambda)$, with the transition between the $\mathrm{RB}$ and the $\mathrm{RX}$ regime being at $\Theta=\Lambda$.
Finally, we rewrite the expression for $\Lambda, \Theta$ (or $\hat{a}$ ), and $\hat{v}$, in Eqs. (1)-(5) using quantities appearing in the driftreduced Braginskii model [Eqs. (7)-(12)] units, with $x \rightarrow \mathrm{R}$ and $y \rightarrow Z$

$$
\begin{gathered}
\Lambda=\frac{\nu n_{1} \mathrm{~L}_{1}^{2}}{\mathrm{~L}_{2} \rho_{\mathrm{s}}}, \\
\Theta=\hat{a}^{\frac{5}{2}}=\left(\frac{a_{b}}{a^{*}}\right)^{\frac{5}{2}}=\left[\frac{\left(\frac{2 a_{\mathrm{Z}}}{\pi}\right)^{4 / 5} a_{\mathrm{R}}^{1 / 5}}{\left(2 \rho_{*}^{-1} \frac{\Delta_{\mathrm{R}} n_{1}}{n_{0,1}} \rho_{\mathrm{s}}^{4} \mathrm{~L}_{2}^{2}\right)^{1 / 5}}\right]^{5 / 2}, \\
\hat{v}=\frac{v_{\mathrm{R}}}{v^{*}}=\frac{v_{\mathrm{R}}}{\left(\rho_{*}^{2} \rho_{\mathrm{s}}^{7} \mathrm{~L}_{2}\right)^{\frac{1}{5}}}\left[8 \frac{\delta n_{1}^{5}}{\Delta_{\mathrm{R}} n_{1}^{2} n_{0,1}^{3}}\left(\frac{\pi a_{\mathrm{R}}}{2 a_{\mathrm{Z}}}\right)^{2}\right]^{-1 / 5} .
\end{gathered}
$$

\section{COMPARISON BETWEEN SIMULATION RESULTS AND ANALYTICAL PREDICTIONS}

Figure 5 (left) locates the detected blobs in the $(\Theta, \Lambda)$ plane. The normalised velocity $\hat{v}$ of each blob as a function of its size $\hat{a}$ is shown in Fig. 5 (right). The detected blobs belong to the RB and $\mathrm{RX}$ regimes, with the threshold between the two regimes being at $\Theta / \Lambda=1$. The analytical scalings of the two-region model for the blob velocity in the RB and RX regimes, traced by continuous blue and red lines, respectively, are shown to be upper bounds of the measured blob velocities. The $\log (\theta / \Lambda)$ colormap indicates whether a blob belongs to the RB or to the RX regime (the RB blobs are blue and the RX blobs are red/yellow). The transition in the color/regime agrees with the change in the velocity trend. We also plot the velocity scaling of the sheath connected, $\mathrm{C}_{\mathrm{s}}$, regime (black dashed line), whose velocity to the size trend is $\hat{a}^{-2}$ (see Fig. 5 left), which differs from the RX scaling

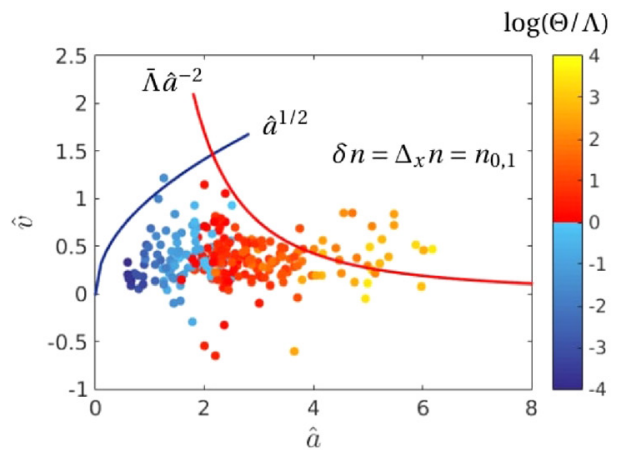

$\log (\Theta / \Lambda)$

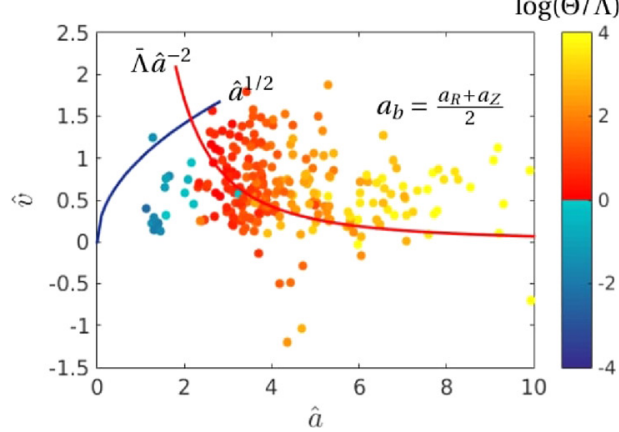

$\log (\Theta / \Lambda)$

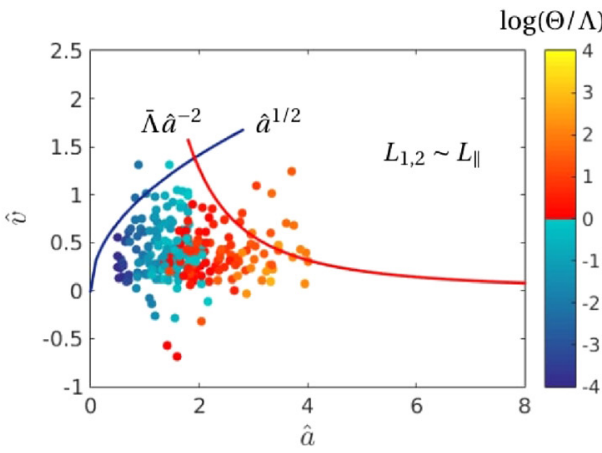

$\log (\Theta / \Lambda)$

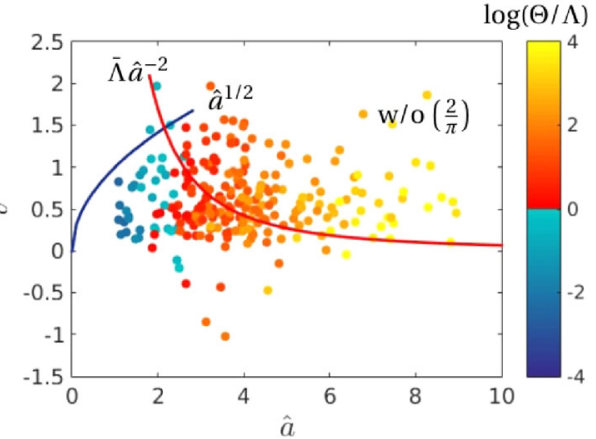

FIG. 6. Effect of various approximations of blob scaling. From left to right, top to bottom, the impact of excluding $\delta \mathrm{n} / \mathrm{n}$ effects, approximating the magnetic field line length in region 1 with $L_{\|}=\left(L_{1}+L_{2}\right)$, considering $a_{b}$ to be the average between the radial blob radius $a_{R}$ and the vertical size $a_{z}$, and removing the $2 / \pi$ factor in $a_{b}$ and $v^{*}$. The qualitative behaviour is similar, but quantitatively the agreement with the analytical scaling is worse than in Fig. 5. 
only by the multiplying factor $\Lambda \sim 10$. In the RX regime, the high collisionality causes the blob to partially disconnect from the sheath, and as a consequence, the blob sustains its self-induced electric field more efficiently, resulting in a faster outwards motion. The simulation results show that the sheath connected scaling significantly underestimates the blob velocity, confirming that the large $a$ blobs belong to the RX regime. To our knowledge, this is the first time that RX behaviour is observed and studied in blob simulations or experiments.

The two-region scaling presented in Sec. II differs to some extent from the one in Myra et $a l^{37}$ as it retains density effects, and the difference between magnetic field line lengths in the upstream and divertor regions, as well as blob ellipticity and the way blob size, is measured. In Fig. 6, we test the influence of these effects on the velocity scaling. The top-left panel of Fig. 6 shows that removing the density perturbation effects shifts the blob distribution to the left and the normalised velocity is reduced. This is due to an increase in both reference size $a^{*}$ and reference velocity $v^{*}$ since we are dropping the terms $\left(\Delta_{x} n_{1} / n_{0,1}\right)^{1 / 5}<1$ and $\left(\delta n_{1}^{5} /\left(\Delta_{x} n_{1}^{2} n_{0,1}^{3}\right)\right)^{1 / 5}<1$ in Eqs. (4) and (6), respectively. Considering the total magnetic field line length from the target to the midplane, $L_{\|}$, rather than the field line length in region $1, \mathrm{~L}_{1}$, (top-right panel of Fig. 6) reduces $\hat{a}$ and impacts the value of $\Lambda$, resulting in a slightly worse agreement between the RB/RX regime transitions, as indicated by the color code and as suggested by the velocity to the size dependence. Finally, taking $a_{b}$ to be the average between $a_{R}$ and $a_{z}$ instead of Eq. (3) (bottom left panel of Fig. 6) significantly impacts the tworegion prediction since most blobs are now estimated to belong to the RX regime, with the blob distribution moving to the right and the normalised size $\hat{a}$ being overestimated. This is partly due, in this case, to the fact that we drop the $(2 / \pi)^{4 / 5}$ term in $a_{b}$, introduced when relating the wavenumber $k_{1}$ in region 1 to $a_{Z}$ (i.e., $\left.k_{1}=2 a_{Z} / \pi\right)$. Removing the $(2 / \pi)^{4 / 5}$ factor contributes to overestimation of the blob size, as shown in the bottom-right panel of Fig. 6.
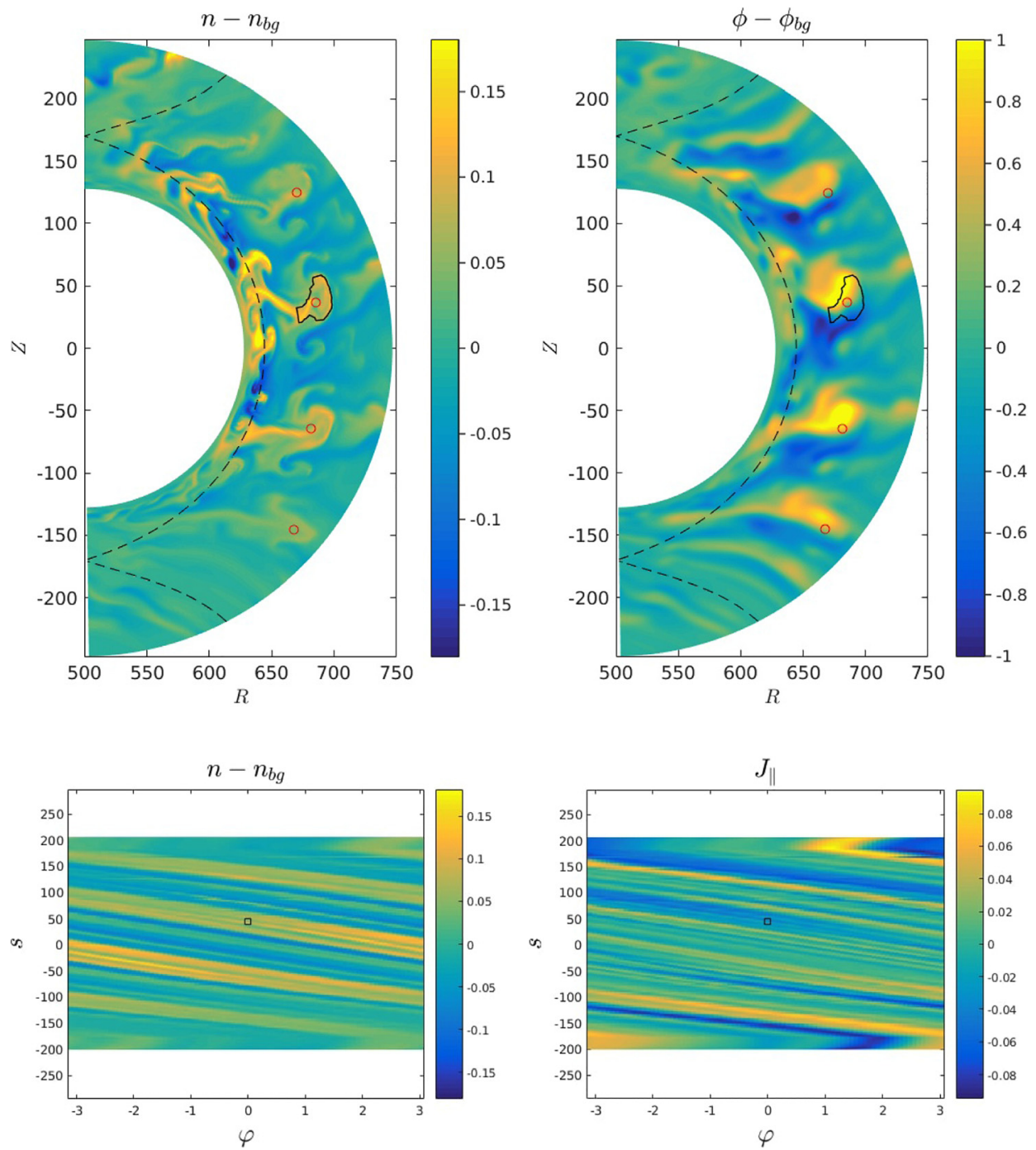

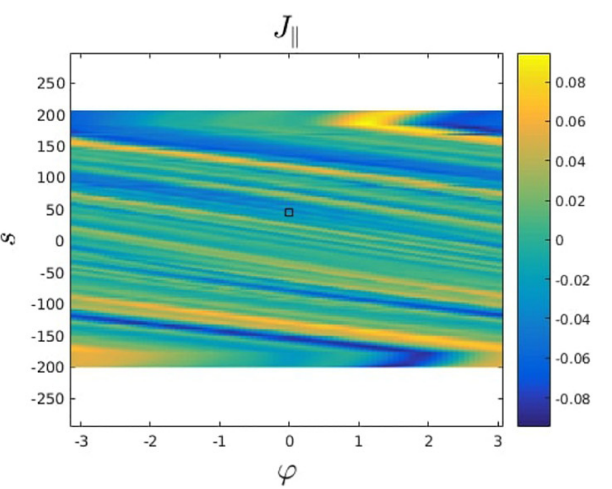

FIG. 7. A resistive-X (RX) blob. From left to right, top to bottom: density fluctuation $n-n_{b g}$ in $(R, Z)$, potential fluctuation $\phi$ $-\phi_{b g}$ in $(R, Z)$ (blob perimeter traced by the continuous black line), density fluctuation along the flux surface, and parallel current $J_{\|}=n\left(v_{\|, i}-v_{\|, e}\right)$ along the flux surface (square indicating the blob center of the mass location). The $\varphi=0$ poloidal plane is represented in the top panels. 
In Figs. 7 and 8, the difference between RB blobs and RX blobs is investigated further by looking at the density, potential, and parallel current of typical blobs belonging to the two regimes. Typically, RB blobs are localised closer to the separatrix, and they do not extend to the divertor region. On the other hand, RX blobs are localised in the far SOL and develop parallel dynamics, reaching the wall. Nonetheless, the associated potential perturbation is relatively small in region 2. A typical blob contoured by a solid black line in the RX regime is shown in Fig. 7. As it can be seen from the top panels, the blob structure extends to the wall and reappears periodically in the poloidal plane, at the locations (identified by red circles) where the magnetic field line that passes through the center-of-mass of the detected blob comes back on the poloidal plane. The blob gets stretched as it approaches the X-points because of the flux expansion present in these regions. The blob elongation along the magnetic field is also confirmed by the bottom panels that show the plasma density and parallel current on the flux surface of the center-of-mass of the blob in the $(s, \varphi)$ plane, where $s$ is the poloidal distance from the midplane, along the magnetic flux surface of the blob, and $\varphi$ is the toroidal angle (the square identifies the blob center-of-mass at $\varphi=0$, which is also shown in the top panels). We remark that the presence of the parallel current in region 1 and region 2 is not negligible. Furthermore, even though the structure can be traced up to the wall, the fluctuations in density and potential decrease moving from the midplane to the wall, indicating partial disconnection of the blob between the sheath and the midplane.

Figure 8 shows the density poloidal snapshot of a RB blob. With respect to the RX blob, it is smaller in size and it is located just outside the separatrix. The electric potential shows the presence of a dipole, which extends outside of the blob perimeter (top right). We note that the blob structure does not reappear periodically on the poloidal plane. Focusing on the flux surface passing through the blob center of mass, we observe that the blob extends along the magnetic field line on the flux
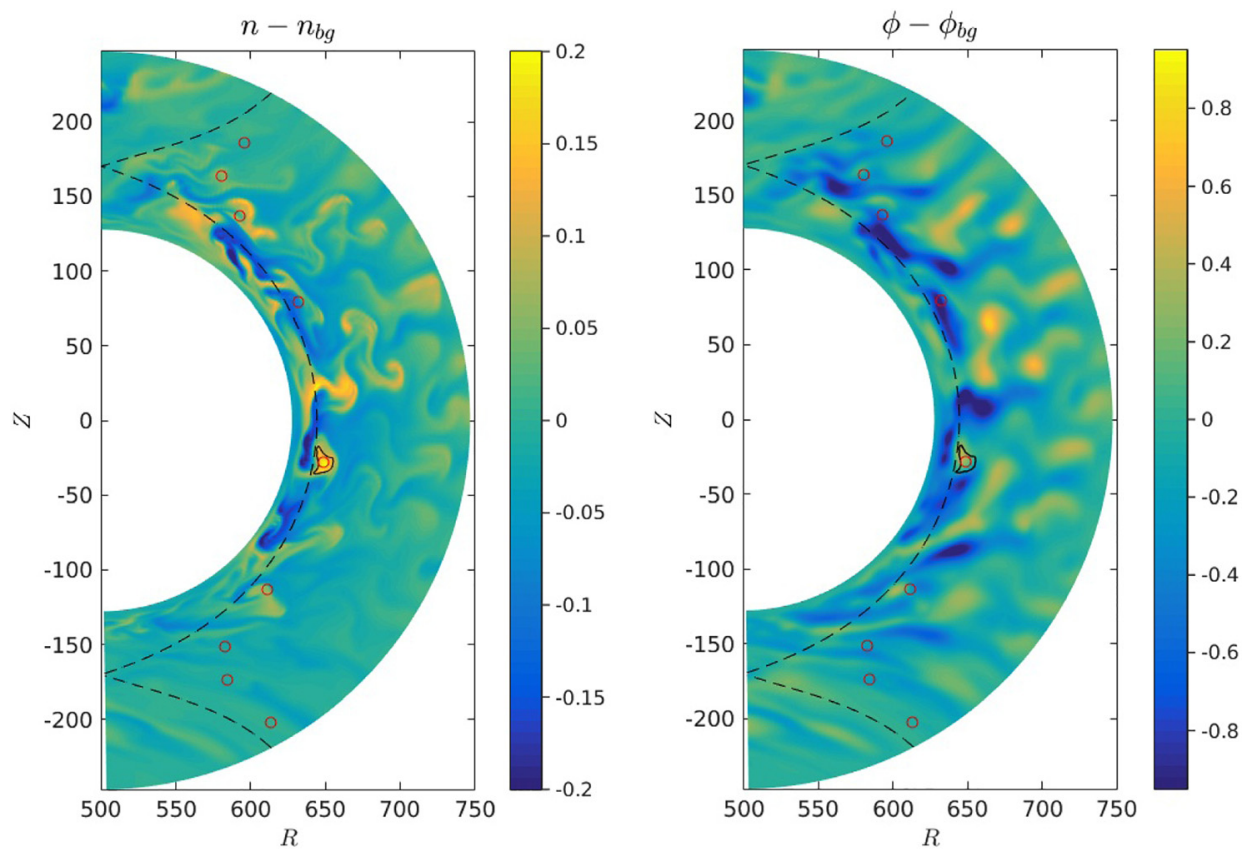

FIG. 8. A resistive ballooning RB blob. From left to right, top to bottom: density fluctuation $n-n_{b g}$ in $(R, Z)$, potential fluctuation $\phi-\phi_{b g}$ in $(R, Z)$ (blob perimeter traced by the continuous black line), density fluctuation along the flux surface, and parallel current $J_{\|}=n\left(v_{\|, i}-v_{\|, e}\right)$ along the flux surface (square indicating the blob center of the mass location). The $\varphi=0$ poloidal plane is represented in the top panels.
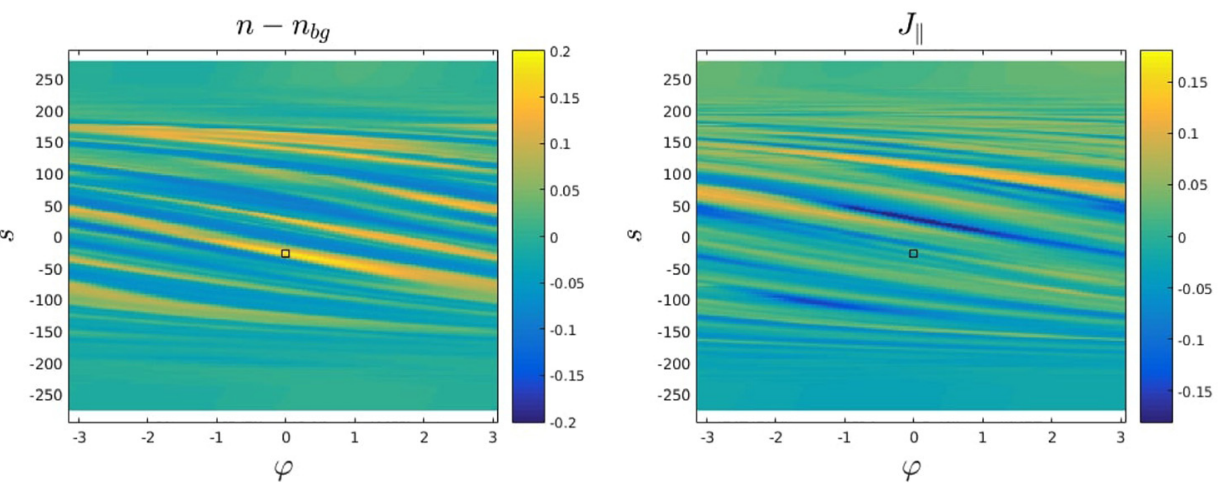
surface, without reaching region 2 that starts at $|s| \geq 150$. Finally, the bottom right plot shows that the parallel current, $\mathrm{J}_{\|}=n\left(v_{\|, i}-v_{\|, e}\right)$, is almost negligible for a RB blob, in agreement with the model that predicts for a RB blob that the curvature drive is compensated by the perpendicular ion-polarisation current, with the parallel dynamics playing a minor role.

We remark that the analytical models used to analyse the results of our simulations are obtained assuming a single blob moving in uniform background fields. Despite this strong assumption, the single blob models appear to be predicting well the simulation results. Indeed, a recent study by Militello et al. ${ }^{45}$ shows that blobs travelling in the SOL can be considered essentially as independent entities. The scattering of the blob velocities with respect to the analytical expectations in Fig. 5 could be due to the non-uniform background where the blob propagates which results from our self-consistent simulations.

\section{CONCLUSIONS}

The GBS code is used to investigate blob dynamics in the presence of an X-point. An analytical scaling for the blob velocity as a function of the blob size and plasma collisionality is derived in a diverted geometry following closely the two-region model presented in Ref. 37. This model accounts for the differences in the physical mechanisms and in the magnetic field geometry that characterise the outboard mid-plane and the divertor regions. The scaling is re-derived starting from a simplified version of the drift-reduced Braginskii's equations for density and vorticity, providing a closure for the parallel dynamics, using Ohm's law and the magnetic pre-sheath physics. The scaling retains the effects of blob plasma density and blob ellipticity, as well as the difference in the magnetic field line length in regions 1 and 2.

A simulation is run in a double-null configuration, and a blob detection/tracking algorithm is developed and used to compute the blob velocity, size, and other physical parameters needed to perform a comparison between simulation results and the analytical scaling. The blobs appear to be in the highcollisionality Resistive Ballooning and Resistive $\mathrm{X}$ regimes of the two-region model, where the curvature drive is balanced by the perpendicular ion polarisation current and parallel current flow between the two regions, respectively. The analytical scaling constitutes an upper bound for the detected blob velocities as a function of their sizes. The effect of blob density and ellipticity is shown to be quantitatively important, although the qualitative trends are unchanged. A detailed analysis of two blobs, one in Resistive $\mathrm{X}$ and one in Resistive Ballooning, shows density and electric potential fluctuations and parallel current profiles that are in agreement with the theoretical expectations. This is the first time that a blob velocity scaling is investigated using full-3D turbulent simulation in diverted geometry.

\section{ACKNOWLEDGMENTS}

We thank Cedric Tsui, James Myra, Federico Nespoli, and Samuel Lanthaler for the useful discussions. The simulations presented herein were carried out in part at the Swiss National Supercomputing Center (CSCS) under Projects ID s718 and s803 and in part on the CINECA Marconi supercomputer under the GBS SOL project. This work was carried out within the framework of the EUROfusion Consortium and received funding from the Fond National Suisse de la Recherche Scientifique and from the Euratom research and training program 2014-2018 under Grant Agreement No. 633053. The views and opinions expressed herein do not necessarily reflect those of the European Commission.

\section{APPENDIX: RE-DERIVATION OF THE TWO REGION MODEL}

The drift-reduced Braginskii's equations for density [Eq. (7)] and vorticity [Eq. (11)] can be simplified around the equatorial midplane as

$$
\begin{aligned}
\frac{\partial \omega_{1}}{\partial \mathrm{t}}+\rho_{*}^{-1}\left[\phi_{1}, \omega_{1}\right] & =\frac{1}{n_{1}} \nabla_{\|} \mathrm{J}_{\|, 1}+\frac{2 \mathrm{~T}_{e}}{n_{1}} \mathrm{C}\left(n_{1}\right) \\
\frac{\partial n_{1}}{\partial \mathrm{t}_{1}}+\rho_{*}^{-1}\left[\phi_{1}, n_{1}\right] & =0
\end{aligned}
$$

and in the divertor region as

$$
\begin{aligned}
\frac{\partial \omega_{2}}{\partial \mathrm{t}}+\rho_{*}^{-1}\left[\phi_{2}, \omega_{2}\right] & =\frac{1}{n_{2}} \nabla_{\|} \mathrm{J}_{\|, 2} \\
\frac{\partial n_{2}}{\partial \mathrm{t}}+\rho_{*}^{-1}\left[\phi_{2}, n_{2}\right] & =0 .
\end{aligned}
$$

With respect to the drift-reduced Braginskii density equation [Eq. (7)], the parallel streaming and magnetic curvature terms are neglected, as they are smaller than the dominant $\mathbf{E} \times \mathbf{B}$ drift. In the vorticity equations, the parallel terms associated with the polarisation current are neglected, and in the divertor region, the interchange drive is also discarded. The large aspect ratio approximation is used, allowing us to drop the normalised magnetic field strength B that appears in Ref. 37.

By balancing the divergence of $J_{\|}$with the resistive term in Ohm's law $\mathrm{J}_{\|}=-\nabla_{\|} \phi / \nu$ in the electron velocity Eq. (8) in the upstream region, we approximate

$$
\nabla_{\|} \mathrm{J}_{\|, 1}=\frac{\phi_{1}-\phi_{2}}{\nu \mathrm{L}_{1}^{2}},
$$

where $L_{1}$ is the length of the magnetic field line from the equatorial midplane to the entrance of the divertor region (normalised to $R_{0}$ ). In the divertor region, a closure for the parallel current can be obtained by integrating $\nabla_{\|} \mathrm{J}_{\|}$along the parallel direction from the interface with the upstream region to the sheath entrance, i.e.,

$$
\int_{2}^{s h} \nabla_{\|} J_{\|, 2} d l=\left.J_{\|}\right|_{2} ^{s h}=-\frac{\phi_{1}-\phi_{2}}{\nu L_{1}}+\frac{n_{2} c_{s}}{T_{e}}\left(\phi_{2}-\phi_{f}\right),
$$

where the sheath current $\mathrm{J}_{\|}=n c_{s}\left(1-\exp \left(\lambda-\phi / \mathrm{T}_{e}\right)\right)$ is linearised around $\phi \sim \phi_{f}=\lambda \mathrm{T}_{e} / e$. By applying the current closures and evaluating the curvature terms at the outboard midplane [using Eq. (16)], the two-region model becomes

$$
\begin{gathered}
\left(\frac{\partial}{\partial \mathrm{t}}+\frac{\mathrm{R}_{0}}{\rho_{\mathrm{s} 0}} \mathbf{v}_{\mathrm{E}, 1} \cdot \nabla\right) \nabla_{\perp}^{2} \phi_{1}=\sigma_{1} \frac{\phi_{1}-\phi_{2}}{n_{1}}-\frac{\beta}{n_{1}} \frac{1}{r} \frac{\partial n_{1}}{\partial \theta}, \\
\left(\frac{\partial}{\partial \mathrm{t}}+\frac{\mathrm{R}_{0}}{\rho_{\mathrm{s} 0}} \mathbf{v}_{\mathrm{E}, 1} \cdot \nabla\right) n_{1}=0,
\end{gathered}
$$




$$
\begin{gathered}
\left(\frac{\partial}{\partial \mathrm{t}}+\frac{\mathrm{R}_{0}}{\rho_{\mathrm{s} 0}} \mathbf{v}_{\mathrm{E}, 2} \cdot \nabla\right) \nabla_{\perp}^{2} \phi_{2}=-\sigma_{2} \frac{\phi_{1}-\phi_{2}}{n_{2}}+\alpha\left(\phi_{2}-\phi_{f}\right), \\
\left(\frac{\partial}{\partial \mathrm{t}}+\frac{\mathrm{R}_{0}}{\rho_{\mathrm{s} 0}} \mathbf{v}_{\mathrm{E}, 2} \cdot \nabla\right) n_{2}=0,
\end{gathered}
$$

having defined

$$
\sigma_{1}=\frac{1}{\nu \mathrm{L}_{1}^{2}}, \quad \sigma_{2}=\frac{1}{\nu \mathrm{L}_{1} \mathrm{~L}_{2}}, \quad \beta=2 \rho_{\mathrm{s}}^{2}, \quad \alpha=\frac{1}{\rho_{\mathrm{s}} \mathrm{L}_{2}},
$$

with $L_{2}$ in the magnetic field line length from the X-point to the wall (in $R_{0}$ units). In addition, in Eqs. (A5)-(A8), the Poisson bracket terms are rewritten as advective terms due to the $\mathbf{E} \times \mathbf{B}$ velocity $\mathbf{v}_{\mathrm{E}}$, for example,

$$
[\phi, \omega]=\mathbf{b} \cdot \nabla \phi \times \nabla \omega=\mathbf{v}_{\mathrm{E}} \cdot \nabla \omega,
$$

where $\mathbf{v}_{\mathrm{E}}$ is dimensionalised over $c_{\mathrm{s} 0}$ and $\nabla$ over $\rho_{\mathrm{s} 0}$.

In order to make analytical progress in the analysis of the blob velocity, we linearise the two-region model. We indicate the radial $\psi$ and binormal $\chi$ directions $\left(\mathbf{e}_{\chi}=\mathbf{b} \times \mathbf{e}_{\psi}\right)$ with $x$ and $y$, respectively (they are normalised to $\rho_{\text {so }}$ units), and Fourier decomposes $\phi_{1,2}$ and $n_{1,2}$ along the $y$ direction, allowing for different wavenumbers in the two regions, i.e.,

$$
\begin{array}{cc}
\phi_{1}=\delta \phi_{1}(x) e^{-i \omega t+i k_{1} y}, & n_{1}=n_{0,1}(x)+\delta n_{1}(x) e^{-i \omega t+i k_{1} y}, \\
\phi_{2}=\delta \phi_{2}(x) e^{-i \omega t+i k_{2} y}, & n_{2}=n_{0,2}(x)+\delta n_{2}(x) e^{-i \omega t+i k_{2} y} .
\end{array}
$$

The background density is given by $n_{b g}=n_{0}-\delta n$ and the peak blob density by $n_{\text {peak }}=n_{0}+\delta n$ (see Fig. 9). We then approximate $\nabla_{\perp}^{2} \delta \phi_{1} \simeq-k_{1}^{2} \delta \phi_{1}$ (assuming the blob electric potential to vary along the $x$ direction on longer scales than along $y$, consistently with the physical picture of a dipole generating in $y$ ), and we work in the $\mathbf{E} \times \mathbf{B}$ frame of reference, so

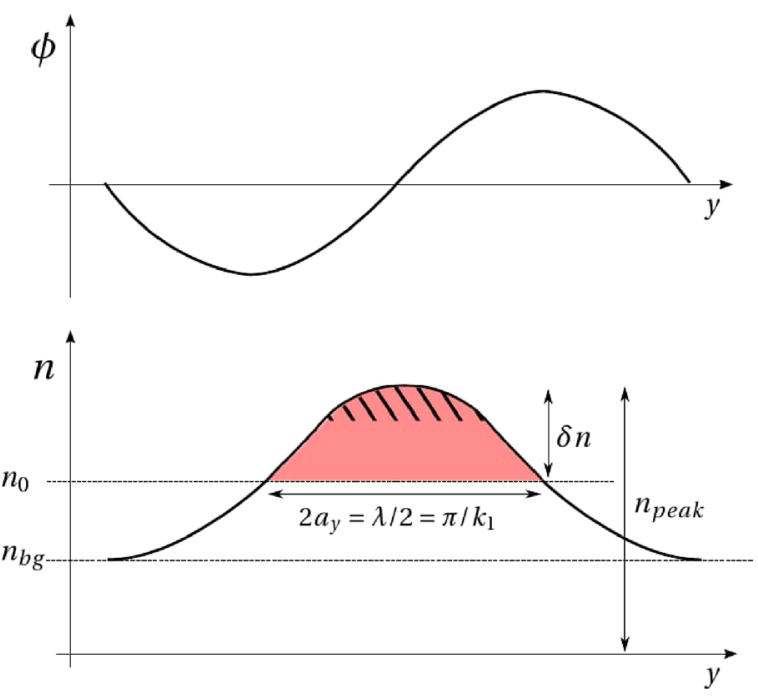

FIG. 9. Blob cut along the vertical direction, $y$, showing potential and density wavelike profiles as expressed in Eqs. (A11) and (A12) and their relation to the blob density peak $n_{\text {peak }}$ and the density background value $n_{\text {bg. }}$. We also show the link between wavenumber $k_{1}$ and half width $a_{y}$ taken at the half maximum location (corresponding to $\left.n=n_{0}\right)$, i.e., $k_{1}=\pi /\left(2 a_{y}\right)$. that the background equilibrium potentials $\phi_{0,1}$ and $\phi_{0,2}$ vanish (assuming that they are constant and equal to each other). The linearized two-region model obtained is the following:

$$
\begin{gathered}
i \omega k_{1}^{2} \delta \phi_{1}=\frac{\sigma_{1}}{n_{0,1}}\left(\delta \phi_{1}-\delta \phi_{2}\right)-i \beta k_{1} \frac{\delta n_{1}}{n_{0,1}}, \\
-i \omega \delta n_{1}-\rho_{*}^{-1} i k_{1} \delta \phi_{1} \frac{\partial n_{1}}{\partial x}=0, \\
i \omega k_{2}^{2} \delta \phi_{2}=\frac{\sigma_{2}}{n_{0,2}}\left(\delta \phi_{2}-\delta \phi_{1}\right)+\alpha \delta \phi_{2}, \\
-i \omega \delta n_{2}-\rho_{*}^{-1} i k_{2} \delta \phi_{2} \frac{\partial n_{2}}{\partial x}=0,
\end{gathered}
$$

where we made use of Eq. (A10) to write $\mathbf{v}_{\mathrm{E}, j} \cdot \nabla \approx-i k_{j} \delta \phi_{j} \partial_{x}$ $\left(j=1\right.$ and 2). Equation (A14) allows us to express $\delta n_{1}$ as a function of $\delta \phi_{1}$, that is,

$$
\delta n_{1}=-\frac{1}{\omega} \rho_{*}^{-1} k_{1} \frac{\partial n_{1}}{\partial x} \delta \phi_{1} .
$$

Note that the background density can vary in the radial direction, $n_{0, j}=n_{0, j}(x)$ in Eqs. (A11) and (A12). In the case of uniform background, $\partial_{x} n_{j}=\partial_{x} \delta n_{j}=\delta n_{j} / a_{x}$, with $a_{x}$ half of the radial blob size. Equation (A17) can be substituted in Eq. (A13) to obtain

$$
\omega^{2} \delta \phi_{1}=-i \frac{\sigma_{1}}{n_{0,1} k_{1}^{2}} \omega\left(\delta \phi_{1}-\delta \phi_{2}\right)+\frac{\beta}{\rho_{*}} \frac{1}{n_{0,1}} \frac{\partial n_{1}}{\partial x} \delta \phi_{1} .
$$

Introducing the characteristic frequencies $\omega_{\sigma, j}=\sigma_{j} /\left(n_{0, j} k_{1}^{2}\right)$ and $\gamma_{\text {mhd }}^{2}=-\beta \rho_{*} \partial_{x} n_{1} / n_{0,1}$, this can be written as

$$
\omega^{2} \delta \phi_{1}=-i \omega_{\sigma, 1} \omega\left(\delta \phi_{1}-\delta \phi_{2}\right)-\gamma_{\mathrm{mhd}}^{2} \delta \phi_{1},
$$

and using the same notation, Eq. (A15) becomes

$$
\omega \delta \phi_{2}=i \omega_{\sigma, 2} \frac{k_{1}^{2}}{k_{2}^{2}}\left(\delta \phi_{1}-\delta \phi_{2}\right)-i \omega_{\alpha, 2} \delta \phi_{2},
$$

where $\omega_{\alpha, 2}=\alpha / k_{2}^{2}$. From Eqs. (A19) and (A20), the following dispersion relation is derived:

$$
\omega^{2}+\gamma_{\mathrm{mhd}}^{2}+\frac{\left(i \omega_{\sigma, 1} \omega\right)\left(\omega+i \omega_{\alpha, 2}\right)}{\omega+i \omega_{\sigma, 2} k_{1}^{2} / k_{2}^{2}+i \omega_{\alpha, 2}}=0 .
$$

Since we can approximate $k_{2}=k_{1} / \varepsilon_{\chi}$, with $\varepsilon_{\chi}$ inversely proportional to the flux tube fanning, Eq. (A21) becomes

$$
\omega^{2}+\gamma_{\text {mhd }}^{2}+\frac{\left(i \omega_{\sigma, 1} \omega\right)\left(\omega+i \varepsilon_{\chi}^{2} \omega_{\alpha, 1}\right)}{\omega+i \varepsilon_{\chi}^{2} \omega_{\sigma, 2}+i \varepsilon_{\chi}^{2} \omega_{\alpha, 1}}=0 .
$$

In Table I, we compare the characteristic frequencies, $\gamma_{\text {mhd }}, \omega_{\sigma, j}$, and $\omega_{\alpha, j}$, with the ones in Myra et al., ${ }^{37}$ in physical units. For an easier comparison, we express our results also in physical units. We note that with the hypothesis of $\tilde{L}_{1}=\tilde{L}_{2}$ and $\tilde{n}_{1}=\tilde{n}_{2}$, our frequency expressions in physical units reduce to the large aspect ratio limit of the ones derived in Ref. 37. For $\omega_{\sigma}$, we use the relation $\nu=e^{2} n_{0} R_{0} /\left(m_{i} \sigma_{\|} c_{s 0}\right)$.

Dividing Eq. (A22) by $\gamma_{\text {mhd }}^{2}$, we obtain

$$
1+\hat{\omega}^{2}+\frac{i \hat{\omega} \Theta\left(\hat{\omega}+i \varepsilon_{\chi}^{2} \Theta\right)}{\Lambda\left(\hat{\omega}+i \varepsilon_{\chi}^{2} \Theta\right)+i \varepsilon_{\chi}^{2} \frac{\omega_{\sigma, 2}}{\omega_{\sigma, 1}} \Theta}=0
$$


TABLE I. Comparison of the characteristic frequencies of the two-region model as derived in the present work and as derived by Myra et al. ${ }^{37}$ In the first column, the dimensionless frequencies are written in GBS dimensionless units, the second column translates them in physical units, and finally, the third column reproduces the expressions from the referenced article. The physical expression $\omega_{\sigma, j}$ is evaluated imposing $\nu=e^{2} n_{0} R_{0} /\left(m_{i} \sigma_{\|} c_{s 0}\right)$, with $\sigma_{\|}$being the parallel conductivity.

\begin{tabular}{|c|c|c|c|}
\hline & $\begin{array}{c}\text { Our model } \\
\text { (Dimensionless units) }\end{array}$ & $\begin{array}{c}\text { Our model } \\
\text { (Physical units) }\end{array}$ & $\begin{array}{l}\text { Myra et al. }{ }^{37} \\
\text { (Physical units) }\end{array}$ \\
\hline$\gamma_{\text {mhd }}^{2}$ & $-2 \rho_{s}^{2} \frac{R_{0}}{\rho_{s 0}} \frac{1}{n_{0,1}} \frac{\partial n_{1}}{\partial x}$ & $-2 \frac{\Omega_{0}^{2} \tilde{\rho}_{s}^{2}}{R_{0}} \frac{1}{\tilde{n}_{0,1}} \frac{\partial \tilde{n}_{1}}{\partial \tilde{x}}$ & $-2 \frac{\tilde{\Omega}^{2} \tilde{\rho}_{s}^{2}}{\tilde{R}} \partial_{\tilde{x}} \ln n_{1}$ \\
\hline$\omega_{\sigma, j}$ & $\left(\nu L_{1} L_{j} n_{0, j} k_{1}^{2}\right)^{-1}$ & $\frac{\Omega_{0}^{2} m_{i} \sigma_{\|}}{e^{2} \tilde{L}_{1} \tilde{L}_{j} \tilde{n}_{0, j} \tilde{k}_{1}^{2}}$ & $\frac{\tilde{\Omega}^{2} m_{i} \sigma_{\|}}{e^{2} \tilde{L}_{\|}^{2} \tilde{n}_{1} \tilde{k}_{1}^{2}}$ \\
\hline$\omega_{\alpha, j}$ & $\left(\rho_{s} L_{2} k_{j}^{2}\right)^{-1}$ & $\frac{\Omega_{0}}{\tilde{\rho}_{s} \tilde{L}_{2} \tilde{k}_{j}^{2}}$ & $\frac{2 \tilde{\Omega}}{\tilde{\rho}_{s} \tilde{L}_{\|} \tilde{k}_{j}^{2}}$ \\
\hline
\end{tabular}

where the normalised frequency $\hat{\omega}=\omega / \gamma_{\text {mhd }}$ is introduced, as well as the parameters that mostly affect the blob motion, i.e., $\Theta=\omega_{\alpha, 1} / \gamma_{\text {mhd }}$ and $\Lambda=\omega_{\alpha, 1} / \omega_{\sigma, 1}$. The $\Theta$ and $\Lambda$ parameters describe the importance of the sheath resistivity with respect to the interchange drive and with respect to the plasma resistivity, respectively. Let us estimate the values of $\Lambda$ and $\Theta$ as functions the blob properties

$$
\begin{gathered}
\Lambda=\frac{n_{1} \alpha}{\sigma_{1}}=\frac{\omega_{\alpha, 1}}{\omega_{\sigma, 1}}=\nu n_{1} \frac{\mathrm{L}_{1}^{2}}{\mathrm{~L}_{2} \rho_{\mathrm{s}}} \\
\Theta=\frac{\omega_{\alpha, 1}}{\gamma_{\mathrm{mhd}}}=\left[\frac{k_{1}^{-4} a_{x}}{2 \rho_{\mathrm{s}}^{4} \mathrm{~L}_{2}^{2} \frac{\Delta_{x} n_{1}}{n_{0,1}} \rho_{*}^{-1}}\right]^{\frac{1}{2}} \stackrel{(2 \mathrm{gg} 9}{=}\left[\frac{\left(2 a_{y} / \pi\right)^{\frac{4}{5}} a_{x}^{\frac{1}{5}}}{\left(2 \rho_{\mathrm{s}}^{4} \mathrm{~L}_{2}^{2} \frac{\Delta_{x} n_{1}}{n_{0,1}} \rho_{*}^{-1}\right)^{\frac{1}{5}}}\right]^{\frac{5}{2}} \\
=\left(\frac{a_{b}}{a^{*}}\right)^{\frac{5}{2}}=\hat{a}^{\frac{5}{2}} .
\end{gathered}
$$

Here, $\Delta_{x} n_{1}$ is an estimate of the variation of the blob density in $x$, such that $\partial_{x} n_{1}$ can be approximated with $\Delta_{x} n_{1} / a_{x}$, where $a_{x}$ represents the blob radius in the radial direction. In the general case of background density varying in $x$ over the blob

TABLE II. Comparison of main blob parameter expression as derived here and as reported in the reference article. ${ }^{37}$ Columns 1 and 2 contain the same expressions in GBS units and in physical units, respectively. Myra's expression in physical units is reported in the third column. In $v^{*}, C(n)=\left(\delta n_{1}^{5} / \Delta_{x} n_{1}^{2} n_{0,1}^{3}\right)\left(\pi a_{x} /\left(2 a_{y}\right)\right)^{2}$.

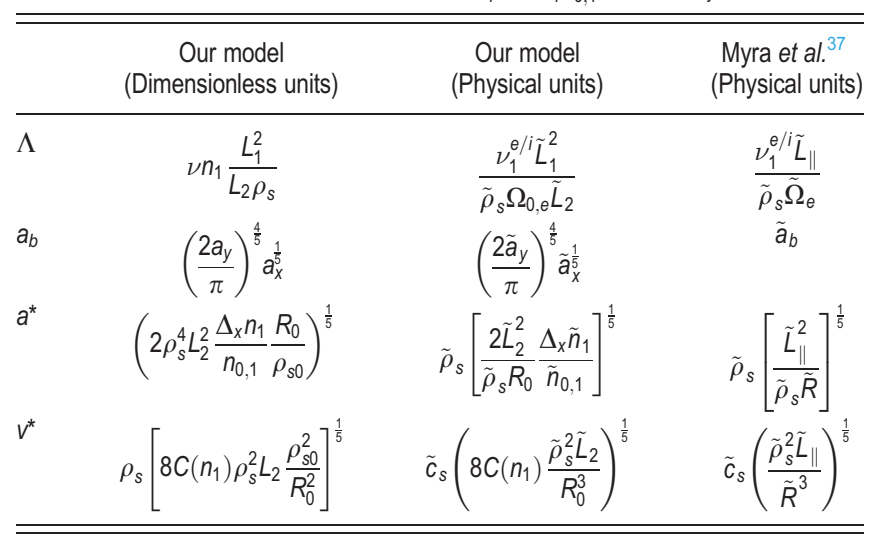

extension, $\Delta_{x} n_{1} \neq \delta n_{1}$ and $a_{x}$ is half of the radial extension of the entire perturbation above the background. In addition, $a_{b}$ $=\left(2 a_{y} / \pi\right)^{4 / 5} a_{x}^{1 / 5}$ is used to estimate the blob size, while $a^{*}$ is the reference size, which is given by the balance between the curvature drive $\beta$ and the sheath current $\alpha$ [defined in Eq. (A9)]

$$
a^{*}=\left(2 \rho_{\mathrm{s}}^{4} \mathrm{~L}_{2}^{2} \frac{\Delta_{x} n_{1}}{n_{0,1}} \rho_{*}^{-1}\right)^{\frac{1}{5}}=\left(\frac{\beta}{\alpha^{2}} \frac{\Delta_{x} n_{1}}{n_{0,1}} \rho_{*}^{-1}\right)^{\frac{1}{5}} .
$$

We now derive an analytical prediction for the blob radial velocity, as a function of the normalised blob size $\hat{a}$ (or $\Theta$ ) and the collisionality $\Lambda$. As a first step, we express the radial velocity $v_{x}$ as a function of the frequency $\omega$. Since the radial blob motion is due to the $\mathbf{E} \times \mathbf{B}$ drift, using the linearised continuity Eq. (A14), one can write

$$
v_{x}=v_{\mathrm{E}}=\operatorname{Im}(\omega) \frac{\rho_{\mathrm{s} 0}}{\mathrm{R}_{0}} \frac{\delta n_{1}}{\Delta_{x} n_{1}} a_{x}
$$

as $\partial_{x} n_{1}=\Delta_{x} n_{1} / a_{x}$ and $v_{E}=-i k_{1} \delta \phi_{1}$. The reference velocity $v^{*}$ is chosen such that the normalised velocity, $\hat{v}=v_{x} / v^{*}$, reads

$$
\hat{v}=\operatorname{Im}(\hat{\omega}) \hat{a}^{1 / 2}
$$

By using Eqs. (A27) and (A28), the reference velocity reads

$$
\begin{aligned}
v^{*} & =\frac{v_{x}}{\operatorname{Im}(\hat{\omega}) \hat{a}^{-1 / 2}}=\gamma_{\operatorname{mhd}} \frac{\rho_{s 0}}{R_{0}} \frac{\delta n_{1}}{\Delta_{x} n_{1}} a_{x} \hat{a}^{-1 / 2} \\
& =\rho_{s}\left[8 \frac{\delta n_{1}^{5}}{\Delta_{x} n_{1}^{2} n_{0,1}^{3}}\left(\frac{\pi a_{x}}{2 a_{y}}\right)^{2} \rho_{s}^{2} L_{2} \rho_{*}^{2}\right]^{\frac{1}{5}} .
\end{aligned}
$$

We note that the chosen reference velocity can be interpreted as the radial velocity of a blob of size $\hat{a}=1$, when the resistive ballooning is the dominant instability, for which the drive in region 1 is balanced by the inertia in the same region [i.e., the first and last terms in Eq. (A19) $]$ and $\omega_{\mathrm{RB}}=i \gamma_{\mathrm{mhd}}$. The main blob parameter expressions as derived here and as reported in the reference article ${ }^{37}$ are summarised in Table II.

In the high collisionality case $\Lambda \gg 1$, of interest in the present paper, one can incur either in the RB, if $\Lambda \gg \Theta$, or in the RX regime, if $\Lambda \ll \Theta$. Since $\Lambda \gg 1$, then $\omega_{\alpha} \gg \omega_{\sigma}$, and the linearised vorticity equation in region 2 [see Eq. (A20)] reduces to

$$
\omega \simeq i \omega_{\sigma, 2} \frac{\delta \phi_{1}}{\delta \phi_{2}}-i \omega_{\alpha, 2}
$$

Since $\omega_{\sigma, 2}$ is small relatively to $\omega_{\alpha, 2}$, either the parallel current term $i \omega_{\sigma, 2} \delta \phi_{1} / \delta \phi_{2}$ drops completely or alternatively $\delta \phi_{1} \gg \delta \phi_{2}$. In the first case, the two regions are completely disconnected and the perturbation does not extend to region 2 , and therefore, $\delta \phi_{2} \sim 0$, and in region 1 , the inertia balances the drive (RB regime). From Eq. (A19)

$$
\omega^{2} \delta \phi_{1}=-\gamma_{\mathrm{mhd}}^{2} \delta \phi_{1}
$$

which leads to $\hat{\omega}_{\mathrm{RB}}=i$ and $\hat{v}_{\mathrm{RB}}=\hat{a}^{\frac{1}{2}}{ }^{46,47}$ On the other hand, if $\delta \phi_{1} \gg \delta \phi_{2}$ (RX regime), in region 1

$$
\omega^{2}=-i \omega_{\sigma, 1} \omega-\gamma_{\mathrm{mhd}}^{2},
$$

i.e., the parallel current balances the interchange drive 


$$
\hat{\omega}_{\mathrm{RX}}=i \frac{\gamma_{\mathrm{mhd}}}{\omega_{\sigma, 1}}=i \frac{\Lambda}{\Theta}
$$

and $\hat{v}_{\mathrm{RX}}=\Lambda \hat{a}^{-2}$. The transition threshold between the two regimes is at $\Theta=\Lambda$, as it can be observed in Eq. (A32)

$$
\hat{\omega}^{2}+i \frac{\Theta}{\Lambda} \hat{\omega}+1=0 .
$$

If $\Lambda>\Theta$ (RB regime), first and third terms balance, and alternatively, if $\Lambda<\Theta$, the second and the third terms balance (RX regime), and the first term drops since $\left|\hat{\omega}^{2}\right|=(\Lambda / \Theta)^{2} \ll 1$. The same result for these two regimes can be obtained more formally but less intuitively, by taking the limit of the dispersion relation in Eq. (A23) for high values of $\Lambda$ and obtaining directly the above Eq. (A34).

\section{REFERENCES}

${ }^{1}$ D. D'Ippolito, J. Myra, and S. Zweben, "Convective transport by intermittent blob-filaments: Comparison of theory and experiment," Phys. Plasmas 18(6), 060501 (2011).

${ }^{2}$ S. Zweben, "Search for coherent structure within tokamak plasma turbulence," Phys. Fluids 28(3), 974-982 (1985).

${ }^{3}$ J. Terry, S. Zweben, K. Hallatschek, B. LaBombard, R. Maqueda, B. Bai, C. Boswell, M. Greenwald, D. Kopon, W. Nevins et al., "Observations of the turbulence in the scrape-off-layer of Alcator C-mod and comparisons with simulation," Phys. Plasmas 10(5), 1739-1747 (2003).

${ }^{4}$ B. Goncalves, C. Hidalgo, C. Silva, M. Pedrosa, and K. Erents, "Statistical description of the radial structure of turbulence in the JET plasma boundary region," J. Nucl. Mater. 337, 376-380 (2005).

${ }^{5}$ H. Tanaka, N. Ohno, N. Asakura, Y. Tsuji, H. Kawashima, S. Takamura, Y. Uesugi et al., "Statistical analysis of fluctuation characteristics at highand low-field sides in L-mode SOL plasmas of JT-60U," Nucl. Fusion 49(6), 065017 (2009).

${ }^{6}$ N. Fedorczak, J. P. Gunn, P. Ghendrih, P. Monier-Garbet, and A. Pocheau, "Flow generation and intermittent transport in the scrape-off-layer of the tore supra tokamak," J. Nucl. Mater. 390, 368-371 (2009).

${ }^{7}$ O. E. Garcia, J. Horacek, R. Pitts, A. H. Nielsen, W. Fundamenski, V. Naulin, and J. J. Rasmussen, "Fluctuations and transport in the TCV scrape-off layer," Nucl. Fusion 47(7), 667 (2007).

${ }^{8}$ U. Pfeiffer, M. Endler, J. Bleuel, H. Niedermeyer, and G. Theimer, "Density, temperature and potential fluctuation measurements with multiple fast swept Langmuir probes on W7-AS," Contrib. Plasma Phys. 38(S1), 134-144 (1998).

${ }^{9}$ T. Happel, F. Greiner, N. Mahdizadeh, B. Nold, M. Ramisch, and U. Stroth, "Generation of intermittent turbulent events at the transition from closed to open field lines in a toroidal plasma," Phys. Rev. Lett. 102(25), 255001 (2009).

${ }^{10}$ N. Vianello, M. Spolaore, M. Agostini, R. Cavazzana, G. De Masi, E. Martines, B. Momo, P. Scarin, S. Spagnolo, and M. Zuin, "On the statistics and features of turbulent structures in RFX-mod," Plasma Phys. Controlled Fusion 58(4), 044009 (2016).

${ }^{11}$ D. Pace, M. Shi, J. Maggs, G. Morales, and T. Carter, "Exponential frequency spectrum and Lorentzian pulses in magnetized plasmas," Phys. Plasmas 15(12), 122304 (2008).

${ }^{12}$ I. Furno, B. Labit, M. Podestà, A. Fasoli, S. Müller, F. Poli, P. Ricci, C. Theiler, S. Brunner, A. Diallo et al., "Experimental observation of the blobgeneration mechanism from interchange waves in a plasma," Phys. Rev. Lett. 100(5), 055004 (2008).

${ }^{13} \mathrm{~S}$. Krasheninnikov, D. D'ippolito, and J. Myra, "Recent theoretical progress in understanding coherent structures in edge and SOL turbulence," J. Plasma Phys. 74(5), 679-717 (2008).

${ }^{14}$ ITER Physics Expert Group on Divertor, ITER Physics Expert on Group on Divertor Modelling and Database, and ITER Physics Basis Editors, "Chapter 4: Power and particle control," Nucl. Fusion 39(12), 2391 (1999).
${ }^{15}$ D. Carralero, P. Manz, L. Aho-Mantila, G. Birkenmeier, M. Brix, M. Groth, H. Müller, U. Stroth, N. Vianello, E. Wolfrum et al., "Experimental validation of a filament transport model in turbulent magnetized plasmas," Phys. Rev. Lett. 115(21), 215002 (2015).

${ }^{16}$ G. Xu, V. Naulin, W. Fundamenski, C. Hidalgo, J. Alonso, C. Silva, B. Goncalves, A. H. Nielsen, J. J. Rasmussen, S. Krasheninnikov et al., "Blob/ hole formation and zonal-flow generation in the edge plasma of the JET tokamak," Nucl. Fusion 49(9), 092002 (2009).

${ }^{17}$ N. Bisai, A. Das, S. Deshpande, R. Jha, P. Kaw, A. Sen, and R. Singh, "Edge and scrape-off layer tokamak plasma turbulence simulation using twofield fluid model," Phys. Plasmas 12(7), 072520 (2005).

${ }^{18}$ C. Theiler, I. Furno, P. Ricci, A. Fasoli, B. Labit, S. Müller, and G. Plyushchev, "Cross-field motion of plasma blobs in an open magnetic field line configuration," Phys. Rev. Lett. 103(6), 065001 (2009).

${ }^{19}$ F. Avino, A. Fasoli, I. Furno, P. Ricci, and C. Theiler, "X-point effect on plasma blob dynamics," Phys. Rev. Lett. 116(10), 105001 (2016).

${ }^{20}$ C. Tsui, J. Boedo, J. Myra, B. Duval, B. Labit, C. Theiler, N. Vianello, W. Vijvers, H. Reimerdes, S. Coda et al., "Filamentary velocity scaling validation in the TCV tokamak," Phys. Plasmas 25(7), 072506 (2018).

${ }^{21}$ F. Riva, C. Colin, J. Denis, L. Easy, I. Furno, J. Madsen, F. Militello, V. Naulin, A. H. Nielsen, J. M. B. Olsen et al., "Blob dynamics in the TORPEX experiment: A multi-code validation," Plasma Phys. Controlled Fusion 58(4), 044005 (2016).

${ }^{22}$ F. Militello, P. Tamain, W. Fundamenski, A. Kirk, V. Naulin, A. H. Nielsen et al., "Experimental and numerical characterization of the turbulence in the scrape-off layer of MAST," Plasma Phys. Controlled Fusion 55(2), 025005 (2013).

${ }^{23}$ J. R. Angus and M. V. Umansky, "Modeling of large amplitude plasma blobs in three-dimensions," Phys. Plasmas 21(1), 012514 (2014).

${ }^{24}$ F. D. Halpern, A. Cardellini, P. Ricci, S. Jolliet, J. Loizu, and A. Mosetto, "Three-dimensional simulations of blob dynamics in a simple magnetized torus," Phys. Plasmas 21(2), 022305 (2014).

${ }^{25}$ F. Nespoli, I. Furno, B. Labit, P. Ricci, F. Avino, F. Halpern, F. Musil, and F. Riva, "Blob properties in full-turbulence simulations of the TCV scrape-off layer," Plasma Phys. Controlled Fusion 59(5), 055009 (2017).

${ }^{26}$ B. W. Shanahan and B. D. Dudson, "Blob dynamics in TORPEX poloidal null configurations," Plasma Phys. Controlled Fusion 58(12), 125003 (2016).

${ }^{27}$ B. Dudson, A. Allen, G. Breyiannis, E. Brugger, J. Buchanan, L. Easy, S. Farley, I. Joseph, M. Kim, A. McGann et al., "Bout++: Recent and current developments," J. Plasma Phys. 81(1), 365810104 (2015).

${ }^{28}$ N. Nace, P. Tamain, C. Baudoin, H. Bufferand, G. Ciraolo, N. Fedorczak, D. Galassi, P. Ghendrih, and E. Serre, "Impact of safety factor and magnetic shear profiles on edge turbulence in circular limited geometry," Contrib. Plasma Phys. 6-8, 497-504 (2018).

${ }^{29}$ W. Gracias, P. Tamain, E. Serre, R. Pitts, and L. Garcia, "The impact of magnetic shear on the dynamics of a seeded 3D filament in slab geometry," Nucl. Mater. Energy 12, 798-807 (2017).

${ }^{30}$ R. Churchill, C. Chang, S. Ku, and J. Dominski, "Pedestal and edge electrostatic turbulence characteristics from an XGC1 gyrokinetic simulation," Plasma Phys. Controlled Fusion 59(10), 105014 (2017).

${ }^{31}$ P. Ricci, F. Halpern, S. Jolliet, J. Loizu, A. Mosetto, A. Fasoli, I. Furno, and C. Theiler, "Simulation of plasma turbulence in scrape-off layer conditions: The GBS code, simulation results and code validation," Plasma Phys. Controlled Fusion 54(12), 124047 (2012).

${ }^{32}$ F. Halpern, P. Ricci, S. Jolliet, J. Loizu, J. Morales, A. Mosetto, F. Musil, F. Riva, T. Tran, and C. Wersal, "The $\{$ GBS $\}$ code for tokamak scrape-off layer simulations," J. Comput. Phys. 315, 388-408 (2016).

${ }^{33}$ P. Paruta, P. Ricci, F. Riva, C. Wersal, C. Beadle, and B. Frei, "Simulation of plasma turbulence in the periphery of diverted tokamak by using the GBS code," Phys. Plasmas 25(11), 112301 (2018).

${ }^{34}$ S. I. Braginskii, "Transport processes in a plasma," Rev. Plasma Phys. 1, 205 (1965).

${ }^{35}$ A. Zeiler, J. F. Drake, and B. Rogers, "Nonlinear reduced Braginskii equations with ion thermal dynamics in toroidal plasma," Phys. Plasmas 4(6), 2134-2138 (1997). 
${ }^{36}$ F. D. Halpern, P. Ricci, B. Labit, I. Furno, S. Jolliet, J. Loizu, A. Mosetto, G. Arnoux, J. Gunn, J. Horacek et al., "Theory-based scaling of the SOL width in circular limited tokamak plasmas," Nucl. Fusion 53(12), 122001 (2013).

37J. Myra, D. Russell, and D. D'Ippolito, "Collisionality and magnetic geometry effects on tokamak edge turbulent transport. I. A two-region model with application to blobs," Phys. Plasmas 13(11), 112502 (2006).

${ }^{38}$ D. Carralero, M. Siccinio, M. Komm, S. Artene, F. D'Isa, J. Adamek, L. AhoMantila, G. Birkenmeier, M. Brix, G. Fuchert et al., "Recent progress towards a quantitative description of filamentary SOL transport," Nucl, Fusion 57(5), 056044 (2017)

${ }^{39}$ L. Easy, F. Militello, J. Omotani, N. Walkden, and B. Dudson, "Investigation of the effect of resistivity on scrape off layer filaments using threedimensional simulations," Phys. Plasmas 23(1), 012512 (2016).

${ }^{40} \mathrm{~S}$. I. Krasheninnikov, "On scrape off layer plasma transport," Phys. Lett. A 283(5-6), 368-370 (2001).

${ }^{41}$ J. Loizu, P. Ricci, F. D. Halpern, and S. Jolliet, "Boundary conditions for plasma fluid models at the magnetic presheath entrance," Phys. Plasmas 19(12), 122307 (2012)

${ }^{42}$ B. LaBombard, A. Kuang, D. Brunner, I. Faust, R. Mumgaard, M. Reinke, J. Terry, J. Hughes, J. Walk, M. Chilenski et al., "High-field side scrape-off layer investigation: Plasma profiles and impurity screening behavior in near-double-null configurations," Nucl. Mater. Energy 12, 139-147 (2017).

${ }^{43}$ B. LaBombard, A. Kuang, D. Brunner, I. Faust, R. Mumgaard, M. Reinke, J. Terry, N. Howard, J. Hughes, M. Chilenski et al., "Impurity screening behavior of the high-field side scrape-off layer in near-double-null configurations: Prospect for mitigating plasma-material interactions on RF actuators and first-wall components," Nucl. Fusion 57(7), 076021 (2017).

${ }^{44}$ C. Theiler, I. Furno, A. Fasoli, P. Ricci, B. Labit, and D. Iraji, "Blob motion and control in simple magnetized plasmas," Phys. Plasmas 18(5), 055901 (2011).

${ }^{45}$ F. Militello, B. Dudson, L. Easy, A. Kirk, and P. Naylor, "On the interaction of scrape off layer filaments," Plasma Phys. Controlled Fusion 59(12), $125013(2017)$

${ }^{46}$ O. Garcia, N. Bian, V. Naulin, A. Nielsen, and J. J. Rasmussen, "Mechanism and scaling for convection of isolated structures in nonuniformly magnetized plasmas," Phys. Plasmas 12(9), 090701 (2005).

47J. Myra and D. D'Ippolito, "Edge instability regimes with applications to blob transport and the quasicoherent mode," Phys. Plasmas 12(9), 092511 (2005). 